\title{
BCS-BEC crossover of ultracold ions driven by density-dependent short-range interactions in a quantum plasma
}

\author{
Ya Zhang, ${ }^{1, *} \mathrm{Yu}$ Wang, ${ }^{1}$ Wei Jiang, ${ }^{2}$ and Weibin $\mathrm{Li}^{3}$ \\ ${ }^{1}$ Department of Physics, Wuhan University of Technology, Wuhan 430070, China \\ ${ }^{2}$ School of Physics, Huazhong University of Science and Technology, Wuhan 430074, China \\ ${ }^{3}$ School of Physics and Astronomy, and Centre for the Mathematics \\ and Theoretical Physics of Quantum Non-Equilibrium Systems, \\ University of Nottingham, NG7 2RD, United Kingdom
}

\begin{abstract}
We study theoretically a novel Bardeen-Cooper-Schrieffer (BCS) to Bose-Einstein condensate (BEC) crossover of two-specie ions in a three-dimensional quantum plasma at zero temperature. Central to this crossover is an effective short-ranged, attractive interaction potential between the ions shielded by the surrounding degenerate electrons. The interaction range and magnitude can be tuned non-monotonically by varying the carrier density of the quantum plasma. Low-energy collisions between two ions are characterized by the s-wave scattering length when the interaction range and the inter-ion spacing are comparable. We show that the s-wave scattering length can be changed from $-\infty$ to $\infty$, leading to a BCS-BEC crossover driven purely by the plasma density. Through numerical and analytical calculations, we find that the quantum acoustic waves in the plasma exhibit distinct dispersion relations in the different regimes, providing a route to probe the crossover. Our study shows that the quantum plasma may offer a new platform to quantum simulate the BEC-BCS crossover and exotic phases with added tunability that might be difficult to achieve in conventional solid-state systems and ultracold atom gases.
\end{abstract}

\section{INTRODUCTION}

In recent years, there is a growing interest in the study of ultracold plasmas, which can be created through photoionizing laser-cooled atoms just above the ionization limit. Shielded by the low-energy electron, the effective two-body interactions between ions are qualitatively different from the bare Coulomb interaction. In particular, a strong coupling regime is obtained when the effective interaction dominates the thermal motions [14]. The Shukla-Eliasson attractive potential between screened ions has been predicted with a quantum hydrodynamic model [5-7], where the plasma density is expected to be similar to the one in the interiors of planets. Importantly the potential can be controlled by the plasma density and temperature, such that the inter-ion separation can be brought down to atomic dimensions [510], additionally due to the quantum statistics [11] and diffraction effects [12]. Previous studies have investigated classical phases, e.g., clustering and crystallization of ions, due to the attractive potential $[6,13,14]$. Densities and spin states of ions constituting the plasmas can be tuned, too. Such controllability has proven to be useful and provides a viable route to manipulate intrinsically different phases of interacting many-body systems. A very successful example is ultracold gases of neutral atoms [15] where the low-energy s-wave scattering can be manipulated by magnetically induced Feshbach resonances between a pair of atoms [16-18]. By varying the s-wave scattering length from $-\infty$ to $+\infty$, a BardeenCooper-Schrieffer (BCS) phase to Bose-Einstein condensate (BEC) crossover is induced [17, 19-33]. Therefore an

*yazhang@whut.edu.cn interesting question is, whether the controllability available in the quantum plasma platform permits to explore quantum many-body states at degenerate temperatures.

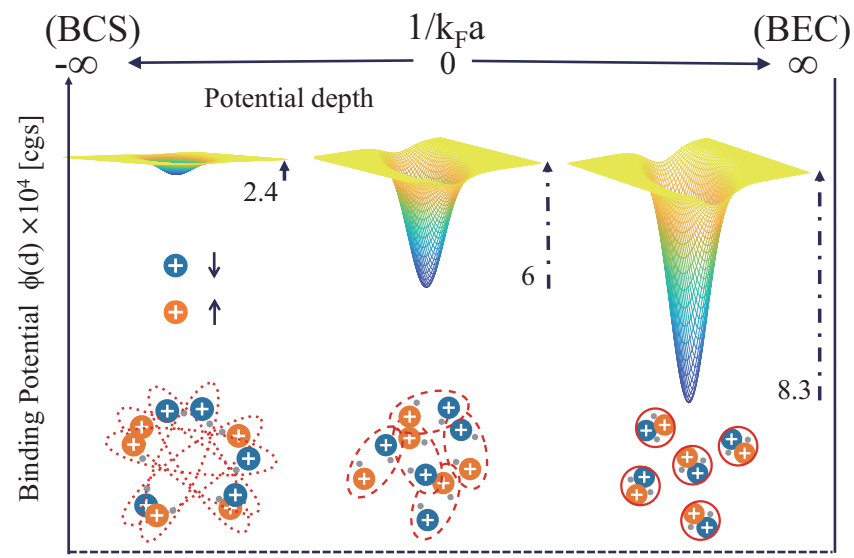

Figure 1. (Color online) Schematic illustration the BCSunitary-BEC regimes. Binding potentials between two ions are shown in the upper row. The depth of the potential depends on the plasma density, which also changes $1 / k_{F} a$ with $k_{F}$ and $a$ to be the Fermi momentum and s-wave scattering length. BCS-BEC crossover of ion pairs is induced by this short-range attractive potential. The orange (blue) spheres represent ${ }^{4} \mathrm{He}^{+}$ions in spin up (down) state and the gray dots are electrons. The Thomas-Fermi screening length of the ion sphere is $1 / q_{s} \approx 0.1 / k_{F}-0.5 / k_{F}$. See text for details.

In this work we study the BCS-BEC crossover of two species (spin up and down) ions in the ultracold quantum plasma. Gauss units are adopted throughout the paper. The screened, short-range attractive potential between ion pairs shielded by surrounding degenerate electrons is a crucial element driving this crossover. As 
shown in Fig. 1, the depth and range of the screened potential can be controlled by modifying densities of the plasma. We show that the inter-ion spacing and range of the screened potential are in the same order of magnitude, such that the s-wave interaction characterizes the interaction between spin-up and spin-down states. The s-wave scattering length can be varied from $-\infty$ to $+\infty$ by changing the plasma density, which plays the role of a magnetic field in the Feshbach resonance of neutral atoms. Consequentially, the ions form Cooper pairs and BEC, determined by vastly different pairing order parameters. The quantum acoustic waves exhibit distinct dispersion relations between unpaired and paired ion configurations through the BCS-BEC crossover. Our study shows that BCS-BEC crossover could be explored in the ultracold quantum plasma in a controlled fashion $[5,34]$. Due to the very high density, the quantum plasma platform may furthermore allow to quantum simulate superfluid, and novel pairing states encountered in nuclear systems $[35,36]$.

\section{THE SCREENED POTENTIAL AND S-WAVE SCATTERING LENGTH}

We consider a quantum plasma consisting of equal density $\left(n_{0}\right)$ degenerate electrons (mass $m_{e}$, charge $-e$ ) and ${ }^{4} \mathrm{He}^{+}$ions (mass $m$, charge $e$ ) at zero temperature, where $e$ is the elementary charge. Due to the screening effect of the electron, the isotropic electric potential $\phi(r)$ [5] between two ions (separation $r=|\mathbf{r}|$ ) is given by

$$
\phi(r)=\frac{e}{2 r}\left[(1+i b) e^{-q_{+} r}+(1-i b) e^{-q_{-r}}\right],
$$

where $q_{ \pm}=\left(q_{s} / \sqrt{4 \alpha}\right)\left[(\sqrt{4 \alpha}+1)^{1 / 2} \pm i(\sqrt{4 \alpha}-1)^{1 / 2}\right]$ and $b=1 / \sqrt{4 \alpha-1}$ in which $\alpha=\hbar^{2} \omega_{e p}^{2} / 4 m_{e}^{2} c_{e 0}^{4}$ and $\omega_{e p}=\sqrt{4 \pi e^{2} n_{0} / m_{e}}$ represent the quantum recoil parameter and electron plasma frequency. Here $c_{e 0}=$ $\sqrt{\frac{n_{0}}{m_{e}} \frac{d^{2}\left[n_{0} \epsilon_{e}\left(n_{0}\right)\right]}{d n_{0}^{2}}}, q_{s}=\omega_{e p} / c_{e 0}, k_{F}=\left(3 \pi^{2} n_{0}\right)^{1 / 3}$ and $\epsilon_{e}\left(n_{0}\right)$ are the sound velocity, inverse Thomas-Fermi screening length, Fermi wave number and ground-state energy of non-interacting electrons, correspondingly. The lengthy but analytical expression of $\epsilon_{e}\left(n_{0}\right)$ is given in Appendix A.

A unique property of the screened potential is that its shape can be changed by varying parameter $\alpha$ through the plasma density, which will be discussed in the following section. For example, the potential has the familiar Thomas-Fermi profile when $\alpha \rightarrow 0$. Crucially important, the landscape of the potential changes dramatically when $\alpha>1 / 4$. A Shukla-Elliason type attractive potential [5$7]$ is obtained, where $\phi(r)$ is negative around the potential minimum at distance $d$, depicted in Fig. 2(a). One can increase the attractive potential depth $\phi(d)$ by decreasing $\alpha$. Note that the electric potential between spin-up and spin-down states and intra-spin states of the ion can be universally described by $\phi(\mathbf{r})$, due to the dressing of the surrounding degenerate electrons in the plasma, as discussed in Appendix A and B.

With the screened potential at hand, we study lowenergy collisions between the spin-up and spin-down species of the ions at zero temperature. The corresponding interaction is captured by the Hamiltonian $[34,37]$,

$$
H=-\frac{\hbar^{2}}{2 m} \nabla^{2}+e \phi+\mu,
$$

where $\nabla^{2}$ is the three dimensional Laplace operator, and $\mu$ is the ion chemical potential.
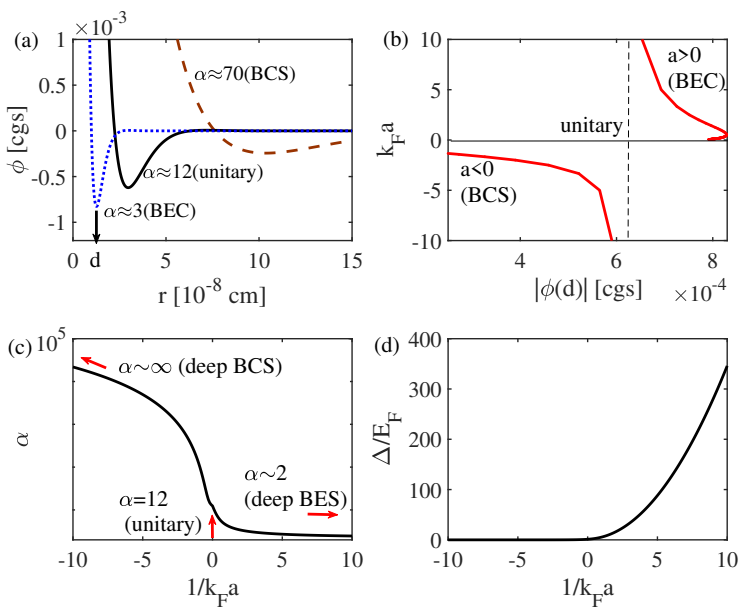

Figure 2. (Color online) Short-range attractive potential and s-wave scattering length. (a) The screened potential $\phi(r)$ is tuned by varying $\alpha$ (through the density $n_{0}=10^{20}, 2 \times$ $10^{22}$, and $10^{24} \mathrm{~cm}^{-3}$, respectively to $\alpha \approx 70,12$, and 3$)$. Decreasing $\alpha$ (increasing $n_{0}$ ), the potential supports the BCS-unitary-BEC crossover. (b) The s-wave scattering length as a function of the binding potential depth, $|\phi(d)|$, with the equilibrium distance $d$ at which the potential takes its minimum value. (c) The dependence of $\alpha$ on the scattering length $a\left(1 / k_{F} a\right)$. (d) The normalized order parameter $\Delta / E_{F}$ as a function of $1 / k_{F} a$.

At zero temperature, the range of the inter-ion potential $\left(\sim 1 / k_{F}\right)$ approaches to the average ion distance $\left(n_{0}^{-1 / 3}\right)$, permitting us to focus on the s-wave scattering between the two spin states [20]. The s-wave scattering length $a$ as a function of the potential depth $\phi(d)$ is shown in Fig. 2(b). Increasing $\phi(d)$, the s-wave scattering length changes from negative to positive. More importantly, the scattering length reaches $\pm \infty$ at around $\phi(d) \approx 6 \times 10^{-4}$. As $\phi(d)$ and $\alpha$ are related, one can also control the scattering length by changing $\alpha$ [Fig. 2(c)], where $1 / k_{F} a$ crosses zero when $\alpha \approx 12$. Such shape is similar to that in cold atomic Fermi gases, where the swave scattering length is modified through, e.g., magnetic Feshbach resonance [23, 38-40]. 


\section{FORMATION OF BCS AND BEC STATES}

The BCS and BEC states due to the long-range part of the Coulomb interaction have been considered in exciton condensates [41]. Inspired by the analogue with Feshbach resonances in cold atomic gases, we will show that spinup and spin-down ion species form Cooper pairs due to the s-wave interaction $[15,18]$. Depending on the scattering length, the order parameter $\Delta$ for Cooper pairs of the spin-up and spin-down ions can be evaluated through [42]

$$
\frac{1}{k_{F} a}=\left(1+\frac{\Delta^{2}}{E_{F}^{2}}\right)^{1 / 4} P_{1 / 2}\left(-1 / \sqrt{1+\frac{\Delta^{2}}{E_{F}^{2}}}\right)
$$

where $P_{1 / 2}(x)$ is the fractional-order Legendre function, and $E_{F}=\hbar^{2} k_{F}^{2} / 2 m$ is the Fermi energy. A non-zero order parameter indicates that a spin-up and a spin-down ion form a pair, such that the plasma is in a BCS state $[15,18]$. As bosonic particles, the Cooper pairs can form a BEC when they are deeply bound, $|\Delta| \gg 1[43]$.

It is necessary to discuss the suitability of Eq. (3) for pairing of fermionic ions in a quantum plasma with a short-range interaction. In this work, it is true that we have focused on the most simple situation where only the s-wave scattering is examined with a short-range interaction like Refs.[20,42]. This is reasonable when the s-wave scattering length $(a)$ is larger or comparable to the range (d) of the inter-particle potential [see Fig. 3(b) below], especially on the BEC side where the order parameter is non-negligible. When the average distance between the quantum ions is larger than the s-wave scattering length, the low-energy scattering will largely not probe the details of the potential at short ranges, which is the requirement that Eq. (3) is a suitable approximation.

The order parameter as a function of $1 / k_{F} a$ is shown in Fig. $2(\mathrm{~d})$. When $1 / k_{F} a$ is negative, $\Delta / E_{F}$ is rather small overall, leading to the BCS region. Increasing $1 / k_{F} a \rightarrow 0$, we enter the so-called unitary regime [18], where $|a|$ is far larger than the average ion spacing, i.e. $n_{0}|a|^{3} \gg 1$. Here $\Delta / E_{F}$ becomes non-negligible, which signatures the formation of a bound state (molecule) of the ion pair [44]. Strikingly, the three dimensional plasma is scale invariance in the unitary regime due to $|a| \rightarrow \infty$, giving rise to conservation laws governed by the continuous symmetry [45]. Further increasing $1 / k_{F} a>0$, the order parameter increases rapidly. The pairs are bound deeply into bosonic molecules, forming a BEC [18]. We emphasize that the different regimes are fully determined by the tunable screened potential. As the examples shown in Fig. 2(a-c), the BEC state of the pairs is achieved through tuning $\alpha \rightarrow 3$ $\left(|\phi(d)|=8.3 \times 10^{-4}\right)$. The unitary regime can be realized when $\alpha \approx 12\left(|\phi(d)| \approx 6 \times 10^{-4}\right)$. We can obtain the BCS of the ions when $\alpha \approx 70\left(|\phi(d)|=2.4 \times 10^{-4}\right)$.

\section{TUNING THE SCREENED POTENTIAL VIA PLASMA DENSITY}

So far, we have identified the dependence of the various states on parameters of the screened potential. In the following, we will show that such dependence can be readily achieved by changing the plasma density $n_{0}$. This density dependence is rooted in the fact that the electron sound velocity $c_{e 0}$ depends nonlinearly on $n_{0}$, which determines the quantum recoil $\alpha$, and substantially the shape of the screened potential. It is found the depth of the screened potential $\phi(d)$ increases with increasing $n_{0}$ [Fig. 3(a)]. Here $\phi(d)$ changes from around $-2.4 \times 10^{-4}$ at $n_{0}=10^{20} \mathrm{~cm}^{-3}$ (BCS) to $8.3 \times 10^{-4}$ at $n_{0}=10^{24} \mathrm{~cm}^{-3}$ (BEC) monotonically, then decrease with further increasing $n_{0}$ to $3 \times 10^{24} \mathrm{~cm}^{-3}$, which is sufficiently large to probe the BCS-BEC crossover [Fig. 2(b)]. By increasing the density, parameter $d$ decreases monotonically. On the BEC side, $d$ is smaller or comparable to the s-wave scattering length (a), as shown in Fig. 3(b). On the other hand, the average spacing between ions is larger than both $d$ and $a$ the BEC and BCS region. For example, the average spacing is $10^{-8} \mathrm{~cm}$ when $n_{0}=10^{24} \mathrm{~cm}^{-3}$ (BEC regime), while $d \approx 10^{-8} \mathrm{~cm}$ and $a \approx 0.6 \times 10^{-8}$ $\mathrm{cm}$. In the BCS region, the average spacing is $2.15 \times 10^{-7}$ $\mathrm{cm}$ when $n_{0}=10^{20} \mathrm{~cm}^{-3}$, where $d \approx 10^{-7} \mathrm{~cm}$, and $a \approx-2 \times 10^{-8} \mathrm{~cm}$. These data are consistent with the assumption, i.e. only considering the s-wave scattering process used in the calculation. This assumption should be a good approximation in the BEC and BCS region at zero temperature.

The main result of this work is that the BCS-unitaryBEC crossover can be obtained by changing the plasma density. We find $\alpha$ can be varied in $2 \sim 70$ when the plasma density changes from $n_{0}=3 \times 10^{24}$ to $10^{20} \mathrm{~cm}^{-3}$ [Fig. 3(c)]. This tunability, in turn, leads to considerable flexibility to control the s-wave scattering length. Our numerical simulations show that the unitary regime is obtained when $n_{0} \approx 2 \times 10^{22} \mathrm{~cm}^{-3}$, where the scattering length $a \rightarrow \pm \infty$, depicted in Fig. 3(d). Away from the unitary regime, the scattering length is finite and negative in the low-density region, such that the ions are in the BCS state. The ions form a degenerate BEC of deeply bound ion pairs [see also Fig. 2(b) and (d)] in a high-density region, where the scattering length is finite and positive. Density-dependent BCS-BEC crossover has predicted in a neutral system at the mean-field level[46]. Our results further show the existence of this crossover in a quantum plasma, which could become a different application field of the BCS-BEC crossover.

We want to point out that the novel BCS-BEC crossover emerges in the strongly coupling regime of the quantum plasma [34, 47-52]. The quantum coupling constant of the screened Coulomb potential is given by the ratio of the average potential to Fermi kinetic energy, i.e. the Brueckner parameter [34], $r_{i} \equiv \frac{1}{k_{F} a_{B}} \propto \frac{|e \phi(d)|}{E_{F}}$, with $a_{B}=\hbar^{2} / e^{2} m$ being the effective Bohr radius. The Brueckner parameter decreases with increasing $n_{0}$, yield- 


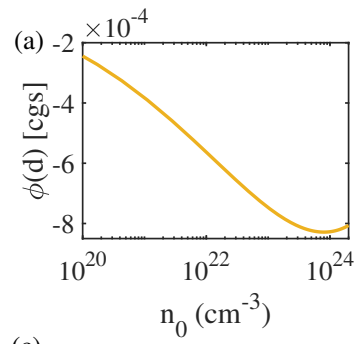

(c)
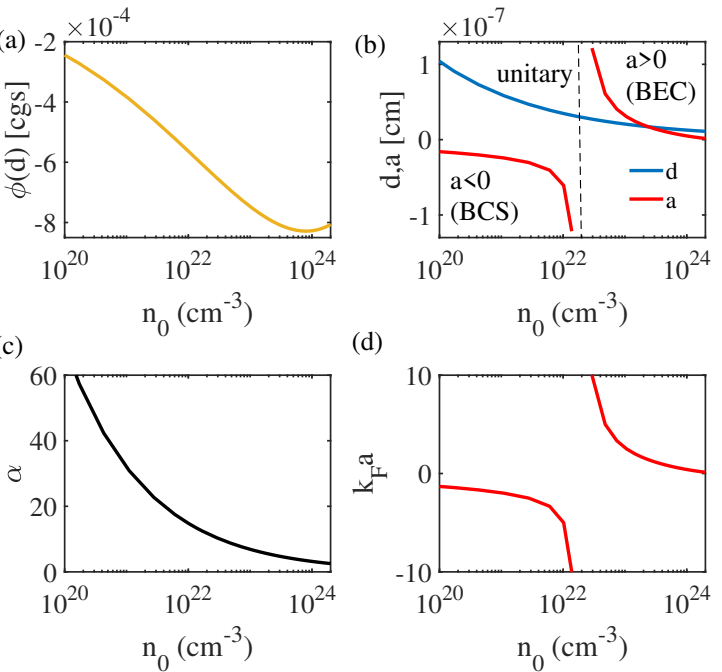

(d)

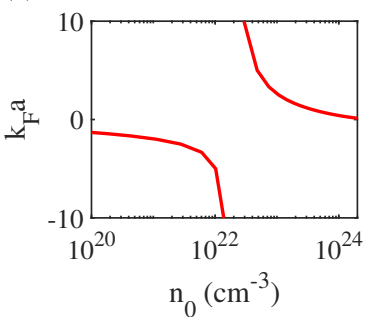

Figure 3. (Color online) Tuning the screened potential via the plasma density. We show (a) the minimum potential $\phi(d)$, (b) the corresponding $d$ compared to the s-wave scattering length $a$, (c) the quantum recoil parameter $\alpha$, and (d) the normalized s-wave scattering length $k_{F} a$ as a function of the plasma density $n_{0}$. The tunability provided by the screened potential allows us to realize the BCS-BEC crossover.

ing $r_{i} \approx 10^{3}-10^{4}$ at $n_{0} \sim 10^{24}-10^{20}$. An interaction-free (ideal) quantum plasma of ions is obtained for $r_{i} \ll 1$ $\left[\frac{|e \phi(d)|}{E_{F}} \ll 1\right]$. The more interesting strong coupling regime is reached around $r_{i} \approx 10^{4}$, which may contain regions of liquid-like and crystalline behavior and bound states [34], where the kinetic energy of the ions plays only a minor role. As shown above, it is in such parameter region that the screened potential gives rise to the $\mathrm{BCS}$, unitary, and BEC states.

\section{STATE DEPENDENT ACOUSTIC WAVES}

Phonon excitation (acoustic wave) exhibits sensitive dependence on the state of the ions (i.e. BCS and BEC). The dispersion relation of the phonon across the BCS$\mathrm{BEC}$ regime can be obtained universally through the kinetic equation formalism [43],

$$
\omega^{2}=\frac{s_{12}^{2}(\omega, q, \mu, \Delta)}{s_{11}(\omega, q, \mu, \Delta) s_{22}(\omega, q, \mu, \Delta)},
$$

where $\omega$ and $q$ are the frequency and wave number in the plasma with pairs. The integrals $s_{11}(\omega, q, \mu, \Delta), s_{22}(\omega, q, \mu, \Delta)$ and $s_{22}(\omega, q, \mu, \Delta)$ enter the linear response function of the plasma [43], whose explicit forms are presented in Appendix A. Eq. (4) shows apparently that the phonon frequency varies with $\Delta$, along with other parameters.

On the other hand, ions with identical spins may not form pairs, but lead to phonon excitations that have dif- ferent dispersion relations, given by

$$
\omega_{0}^{2}=\frac{4 e}{m} \mathcal{L}(d) \sin ^{2}\left(\frac{q d}{2}\right)+c_{i 0}^{2} q^{2}+\frac{\hbar^{2} q^{4}}{4 m^{2}},
$$

with $\mathcal{L}(d)=\left.\frac{d^{2} \phi(r)}{d r^{2}}\right|_{r=d}$ is the radial Laplacian of the screened potential and $c_{i 0}$ is the sound velocity of the ions. In this situation the frequency $\omega_{0}$ depends solely on the density [through $d, c_{i 0}$ and $\left.\mathcal{L}(d)\right]$. It has been theoretically predicted and experimentally justified that, the phonons are found to obey a dispersion relation that assumes a Thomas-Fermi or Yukawa inter-particle potential [53-55]. The Yukawa potential can be given by Eq. (A17) for $\alpha \longrightarrow 0$.
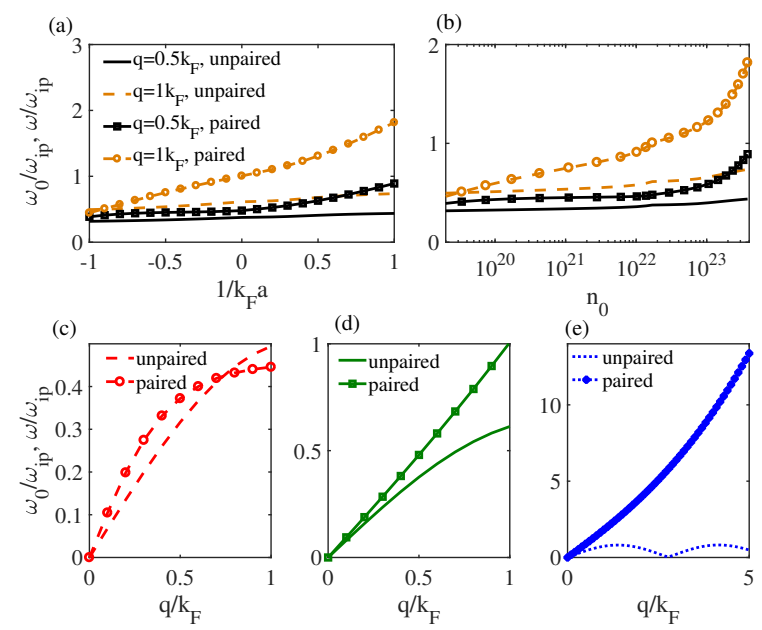

Figure 4. (Color online) Phonon dispersion in different regimes. Phonon frequency $\omega / \omega_{i p}$ and $\omega_{0} / \omega_{i p}$ (normalized by the ion plasma frequency $\omega_{i p}=\sqrt{4 \pi e^{2} n_{0} / m}$ ) when varying (a) $1 / k_{F} a$ and (b) $n_{0}$. It is found that the frequency is higher in the paired plasma. Phonon dispersion in the (c) BCS, (d) unitary and (e) BEC regime. Other parameters are (c) $1 / k_{F} a=-1$ $\left(n_{0}=2 \times 10^{19} \mathrm{~cm}^{-3}\right)$, (d) $1 / k_{F} a=0\left(n_{0}=2 \times 10^{22} \mathrm{~cm}^{-3}\right)$, and (e) $1 / k_{F} a=1\left(n_{0}=4 \times 10^{23} \mathrm{~cm}^{-3}\right)$.

Phonon frequencies at given wavenumber $q$ are displayed in Fig. 4(a) and (b) by varying the scattering length and density, correspondingly. Both $\omega$ and $\omega_{0}$ depend on $1 / k_{F} a$ as well as plasma density $n_{0}$ nonlinearly. At a given $q$, a general feature is $\omega>\omega_{0}$, as shown in the figure. Phonon excitation can be achieved with laser or ion beam compression [56], providing a way to experimentally measure the state and density-dependent acoustic waves. In the following, we examine the dispersion of the phonon mode at different scattering lengths (density) in detail. On the BCS side $(a<0), \omega$ increases linearly with $q$ when $q$ is small, and approaches $2 \Delta$ in the pairbreaking continuum limit [43] when $q>q_{c}=2 \sqrt{2 m \mu}$, depicted in Fig. 4(c). As scattering length $a$ is fixed, the order parameter $\Delta$ is a constant, giving the maximal frequency $\omega_{\max }=2 \Delta$. In this limit, the presence of the Fermi sea of the quantum ions makes the formation of pairs difficult due to the Pauli principle. The 
behavior in the dispersion relation is a unique feature of the ultracold quantum plasma since the paired fermions interact through the short-range, screened potential instead of the long-range Coulomb potential. Here $\omega_{0}$ is slightly smaller than $\omega$ at low momentum due to the weak but non-negligible order parameter. Further increasing $q>q_{c}$, we find $\omega_{0}$ becomes larger. Different from $\omega \rightarrow 2 \Delta$ in the pair-breaking regime, $\omega_{0}$ does not have an upper bound and increases quadratically with increasing $q$ due to the quantum pressure of ions [see the last term in Eq. (5)].

At the unitary $\left(1 /\left|k_{F} a\right|=0\right)$, phonon frequencies of the paired ions increase almost linearly with increasing $q$ [Fig. 4(d)], similar to ideal phonon modes. It will eventually reach the pair-breaking limit (not shown in the figure). In comparison, $\omega_{0}$ is always smaller than $\omega$ when $q / k_{F}<1$. Though $\omega_{0}$ becomes larger due to the quadratic term in Eq. (5), $\omega$ increases more rapidly with $q$, and succeeds $\omega_{0}$ considerably at high momentum region. Deep in the BEC regime, $\omega$ linearly increases with $q$ when $q \ll k_{F}$. However, the dispersion is quadratic concerning $q$ at a larger value, as shown in Fig. 4(e). The excitation spectra are similar to the one found in molecular condensates, where these paired ions behave as elementary bosons [22]. In comparison, $\omega_{0}$ is sinusoidal and always smaller than $\omega$ when $q / k_{F} \leq 5$. The dependence of phonon dispersions on the scatterring length, qualitatively agrees with that reported in Ref.[57] within the random-phase approximation method in the field of cold atoms. Thus, our result contributes to expanding the existing knowledge of BCS-BEC crossover from atomic systems to positive ion systems.

The above analysis shows that the formation of ion pairs can alter the dispersion relation of the phonons, which provides a feasible route for experimental test on this specific kind of BCS-BEC crossover. The dispersion relations of phonon excitations could be experimentally measured using the laser-excitation method introduced by Nunomura et. al $[54,55]$. In their experiments, particles are imaged by a charge-coupled device video camera. Images are recorded on a videocassette recorder tape at 30 frames per second, where each particle's coordinates can be identified. The real and imaginary parts of the wave vector have been obtained by fitting the phase shift and amplitude decay of the waves as functions of particle distance from analyzing particle motions. Their experimental results agree well with theory [53] applicable to strongly coupled screened Coulomb crystals with a Thomas-Fermi or Yukawa potential.

\section{CONCLUSIONS}

We have studied the BCS-BEC crossover in an ultracold ion plasma interacting through a short-range, attrac- tive screened potential arising due to the collective quantum behavior of the surrounding electron. The plasma density can control the depth and range of the screened potential. Focusing on a regime where the mean ion separation and the characteristic range of the potential are on the same order of magnitude, the s-wave scattering length between two different spin states of the ions can be varied from $-\infty$ to $+\infty$. We have shown that this allows the formation of BCS, unitary, and BEC states of ions in different spin states. Significantly the phonon excitation in the plasma strongly depends on the order parameter $\Delta$, offering a route to probe the presence of the Cooper pairs. Our study reveals a novel mechanism to achieve the BCS-BEC crossover in ultracold quantum plasma, complimenting the Feshbach resonance typically employed in ultracold gases of neutral atoms. Our study might open a new window to explore many-body physics, scale invariance as well as carry out quantum simulation of superfluid states of nuclear matters with the ultracold, quantum plasma [2-4].

In the present study, we have focused on the s-wave scattering of the quantum plasma at zero temperature. When the density of the plasma is high, i.e. deep in the BEC regime, it might become necessary to consider higher partial waves e.g. p-wave scattering [58, 59], as the two-body collision could be affected by the structure of the potential at short distances. In this case, the simple treatment (i.e. pairing order parameter obtained from Eq. (3)) will not be sufficient. One can explore the underlying phases using BCS theory that taking into account of non-zero momentum contributions [60,61]. The BCS theory provides a mean-field framework to not only describe the s-wave and p-wave pairing, but also to study phases at finite temperatures [17]. The respective phases and their dependence on the parameter of the electric potential are worth to pursuing in the future.

\section{ACKNOWLEDGMENTS}

This work was supported by the Natural Science Foundation of China through Grants Nos. 11975174 and 11775090. W. L. acknowledges support from the EPSRC through Grant No. EP/R04340X/1 via the QuantERA project "ERyQSenS," the UKIERI-UGC Thematic Partnership (IND/CONT/G/16-17/73), and the Royal Society through the International Exchanges Cost Share award No. IEC $\backslash$ NSFC $\backslash 181078$. The authors thank Kai Liao for helpful suggestions. 
[1] T. C. Killian, S. Kulin, S. D. Bergeson, L. A. Orozco, C. Orzel, and S. L. Rolston, Physical Review Letters 83, 4776 (1999).

[2] T. C. Killian, Science 316, 705 (2007).

[3] M. Lyon and S. L. Rolston, Reports on Progress in Physics 80, 017001 (2016).

[4] T. K. Langin, G. M. Gorman, and T. C. Killian, Science 363, 61 (2019).

[5] P. K. Shukla and B. Eliasson, Physical Review Letters 108, 165007 (2012).

[6] P. K. Shukla and B. Eliasson, Journal of Plasma Physics 79, 359 (2013).

[7] P. K. Shukla, B. Eliasson, and M. Akbari-Moghanjoughi, Physical Review E 87, 037101 (2013).

[8] M. Akbari-Moghanjoughi, Journal of Plasma Physics 79, 189 (2013).

[9] H. Hu, L. Li, Z. Chen, W. Chen, X. Liu, and P. Li, Physics of Plasmas 26, 082108 (2019).

[10] Z. Moldabekov, T. Dornheim, and M. Bonitz, arXiv:2009.09180 [physics] (2020).

[11] P. K. Shukla and B. Eliasson, Reviews of Modern Physics 83, 885 (2011).

[12] J. T. Mendonça, Physics of Plasmas 18, 062101 (2011).

[13] M. Bonitz, V. S. Filinov, V. E. Fortov, P. R. Levashov, and H. Fehske, Physical Review Letters 95, 235006 (2005).

[14] S. V. Vladimirov and O. Ishihara, Physics of Plasmas 3, 444 (1996).

[15] C. J. Pethick and H. Smith, Bose-Einstein Condensation in Dilute Gases (2008).

[16] M. Greiner, C. A. Regal, and D. S. Jin, Physical Review Letters 94, 070403 (2005).

[17] S. Giorgini, L. P. Pitaevskii, and S. Stringari, Reviews of Modern Physics 80, 1215 (2008).

[18] C. Chin, R. Grimm, P. Julienne, and E. Tiesinga, Reviews of Modern Physics 82, 1225 (2010).

[19] M. H. Anderson, J. R. Ensher, M. R. Matthews, C. E. Wieman, and E. A. Cornell, Science 269, 198 (1995).

[20] C. C. Bradley, C. A. Sackett, J. J. Tollett, and R. G. Hulet, Physical Review Letters 75, 1687 (1995).

[21] C. A. Regal, M. Greiner, and D. S. Jin, Physical Review Letters 92, 040403 (2004).

[22] M. W. Zwierlein, C. A. Stan, C. H. Schunck, S. M. F. Raupach, A. J. Kerman, and W. Ketterle, Physical Review Letters 92, 120403 (2004).

[23] M. Bartenstein, A. Altmeyer, S. Riedl, S. Jochim, C. Chin, J. H. Denschlag, and R. Grimm, Physical Review Letters 92, 203201 (2004).

[24] T. Bourdel, L. Khaykovich, J. Cubizolles, J. Zhang, F. Chevy, M. Teichmann, L. Tarruell, S. J. J. M. F. Kokkelmans, and C. Salomon, Physical Review Letters 93, 050401 (2004).

[25] E. Hodby, S. T. Thompson, C. A. Regal, M. Greiner, A. C. Wilson, D. S. Jin, E. A. Cornell, and C. E. Wieman, Physical Review Letters 94, 120402 (2005).

[26] M. Schemmer, I. Bouchoule, B. Doyon, and J. Dubail, Physical Review Letters 122, 090601 (2019).

[27] B. Mukherjee, P. B. Patel, Z. Yan, R. J. Fletcher, J. Struck, and M. W. Zwierlein, Physical Review Letters 122, 203402 (2019).

[28] D. M. Eagles, Physical Review 186, 456 (1969).
[29] D. S. Petrov, C. Salomon, and G. V. Shlyapnikov, Physical Review Letters 93, 090404 (2004).

[30] L. Salasnich and F. Toigo, Physical Review A 78, 053626 (2008).

[31] Y. E. Kim and A. L. Zubarev, Physical Review A 72, 011603 (2005).

[32] N. Manini and L. Salasnich, Physical Review A 71, 033625 (2005).

[33] M. K. Tey, L. A. Sidorenkov, E. R. S. Guajardo, R. Grimm, M. J. H. Ku, M. W. Zwierlein, Y.-H. Hou, L. Pitaevskii, and S. Stringari, Physical Review Letters 110, 055303 (2013).

[34] M. Bonitz, N. Horing, and P. Ludwig, Introduction to Complex Plasmas (Springer Science \& Business Media, 2010).

[35] G. C. Strinati, P. Pieri, G. Röpke, P. Schuck, and M. Urban, Physics Reports 738, 1 (2018).

[36] Y. Ohashi, H. Tajima, and P. van Wyk, Prog. Part. and Nucl. Phys. 111, 103739 (2020).

[37] G. Manfredi and F. Haas, Physical Review B 64, 075316 (2001).

[38] T. Stöferle, H. Moritz, K. Günter, M. Köhl, and T. Esslinger, Physical Review Letters 96, 030401 (2006).

[39] M. Randeria and E. Taylor, Ann. Rev. Cond. Matt. Phys. 5, 209 (2014).

[40] J. N. Fuchs, A. Recati, and W. Zwerger, Physical Review Letters 93, 090408 (2004).

[41] A. Griffin, D. W. Snoke, and S. Stringari, Bose-Einstein Condensation (Cambridge University Press, 1996).

[42] T. Papenbrock and G. F. Bertsch, Physical Review C 59, 2052 (1999).

[43] R. Combescot, M. Y. Kagan, and S. Stringari, Physical Review A 74, 042717 (2006).

[44] A. Bulgac, J. E. Drut, and P. Magierski, Physical Review Letters 96, 090404 (2006).

[45] M. J. H. Ku, A. T. Sommer, L. W. Cheuk, and M. W. Zwierlein, Science 335, 563 (2012).

[46] N. Andrenacci, A. Perali, P. Pieri, and G. C. Strinati, Physical Review B 60, 12410 (1999), publisher: American Physical Society.

[47] V. Fortov, I. Iakubov, and A. Khrapak, Physics of Strongly Coupled Plasma (OUP Oxford, 2006).

[48] Z. A. Moldabekov, S. Groth, T. Dornheim, H. Kählert, M. Bonitz, and T. S. Ramazanov, Physical Review E 98, 023207 (2018).

[49] Z. A. Moldabekov, H. Kählert, T. Dornheim, S. Groth, M. Bonitz, and T. S. Ramazanov, Physical Review E 99, 053203 (2019).

[50] S. Ghosh, N. Chakrabarti, and P. K. Shukla, Physics of Plasmas 19, 072123 (2012).

[51] R. P. Drake, High-Energy-Density Physics: Foundation of Inertial Fusion and Experimental Astrophysics (Springer, 2018).

[52] P. K. Shukla and B. Eliasson, Physics-Uspekhi 53, 51 (2010).

[53] X. Wang, A. Bhattacharjee, and S. Hu, Physical Review Letters 86, 2569 (2001), publisher: American Physical Society.

[54] S. Nunomura, J. Goree, S. Hu, X. Wang, and A. Bhattacharjee, Physical Review E 65, 066402 (2002), publisher: American Physical Society. 
[55] S. Nunomura, D. Samsonov, and J. Goree, Physical Review Letters 84, 5141 (2000), publisher: American Physical Society.

[56] E. G. Gamaly and A. V. Rode, Progress in Quantum Electronics 37, 215 (2013).

[57] S. Van Loon, W. Van Alphen, J. Tempere, and H. Kurkjian, Physical Review A 98, 063627 (2018), publisher: American Physical Society.

[58] J. L. Bohn, Physical Review A 61, 053409 (2000), publisher: American Physical Society.

[59] C. A. Regal, C. Ticknor, J. L. Bohn, and D. S. Jin, Physical Review Letters 90, 053201 (2003).

[60] T.-L. Ho and R. B. Diener, Phys. Rev. Lett. 94, 090402 (2005).

[61] V. Gurarie, L. Radzihovsky, and A. V. Andreev, Phys. Rev. Lett. 94, 230403 (2005).

[62] Y. E. Kim and A. L. Zubarev, Physical Review A 70, 033612 (2004).

[63] L. Kai, C. Ji-Sheng, and L. Chao, Communications in Theoretical Physics 54, 489 (2010).

[64] N. Crouseilles, P.-A. Hervieux, and G. Manfredi, Physical Review B 78, 155412 (2008).

[65] L. Wei and Y.-N. Wang, Physical Review B 75, 193407 (2007).

[66] F. Haas, Quantum Plasmas: An Hydrodynamic Approach (Springer Science \& Business Media, 2011).

[67] M. Marklund and G. Brodin, Physical Review Letters 98, 025001 (2007).

[68] Y. Zhang, F. Zhai, B. Guo, L. Yi, and W. Jiang, Physical Review B 96, 045104 (2017).

[69] J. A. Frenje, P. E. Grabowski, C. K. Li, F. H. Séguin, A. B. Zylstra, M. Gatu Johnson, R. D. Petrasso, V. Y. Glebov, and T. C. Sangster, Physical Review Letters 115, 205001 (2015).

[70] S. V. Vladimirov and Y. O. Tyshetskiy, Physics-Uspekhi 54, 1243 (2011).

[71] Y. E. Kim and A. L. Zubarev, J. of Phys. B 38, L243 (2005).

[72] C. Kittel and P. McEuen, Introduction to solid state physics, Vol. 8 (Wiley New York, 1976).

[73] C. A. R. Sá de Melo, M. Randeria, and J. R. Engelbrecht, Physical Review Letters 71, 3202 (1993).

[74] L. Pitaevskii and S. Stringari, Physical Review Letters 81, 4541 (1998).

[75] W. Dappen and A. Nayfonov, Astrophysical J. Supp. Seri. 127, 287 (2000).

[76] G. Chabrier, The Astrophysical Journal 414, 695 (1993).

[77] A. Y. Potekhin, G. Chabrier, and D. G. Yakovlev, Contributions to Plasma Physics 41, 231 (2001).

[78] V. B. Mintsev and V. E. Fortov, J. Phys. A 39, 4319 (2006).

[79] E. Boggasch, J. Jacoby, H. Wahl, K.-G. Dietrich, D. H. H. Hoffmann, W. Laux, M. Elfers, C. R. Haas, V. P. Dubenkov, and A. A. Golubev, Physical Review Letters 66, 1705 (1991).

[80] M. K. Matzen, C. Deeney, R. J. Leeper, J. L. Porter, R. B. Spielman, G. A. Chandler, M. S. Derzon, M. R. Douglas, D. L. Fehl, D. E. Hebron, T. J. Nash, R. E. Olson, L. E. Ruggles, T. W. L. Sanford, J. F. Seamen, K. W. Struve, W. A. Stygar, and D. L. Peterson, Plasma Physics and Controlled Fusion 41, A175 (1999).

[81] H. J. Kusch, J. Phys. E 18, 654 (1985).

[82] M. D. Perry and G. Mourou, Science 264, 917 (1994), tex.copyright: 1994 by the American Association for the
Advancement of Science.

[83] M. Roth, T. E. Cowan, M. H. Key, S. P. Hatchett, C. Brown, W. Fountain, J. Johnson, D. M. Pennington, R. A. Snavely, S. C. Wilks, K. Yasuike, H. Ruhl, F. Pegoraro, S. V. Bulanov, E. M. Campbell, M. D. Perry, and H. Powell, Physical Review Letters 86, 436 (2001).

[84] M. Hofmann, M. Bleicher, S. Scherer, L. Neise, H. Stöcker, and W. Greiner, Physics Letters B 478, 161 (2000).

[85] A. Peshier, B. Kämpfer, and G. Soff, Physical Review D 66, 094003 (2002).

[86] B. A. Gelman, E. V. Shuryak, and I. Zahed, Physical Review C 74, 044908 (2006).

[87] N. Rynn and N. D'Angelo, Review of Scientific Instruments 31, 1326 (1960).

[88] A. Y. Wong, R. W. Motley, and N. D'Angelo, Physical Review 133, A436 (1964).

[89] R. Yokogawa, H. Takeuchi, Y. Arai, I. Yonenaga, M. Tomita, H. Uchiyama, T. Watanabe, and A. Ogura, Appl. Phys. Lett. 116, 242104 (2020), publisher: American Institute of Physics.

[90] J. Bardeen, L. N. Cooper, and J. R. Schrieffer, Physical Review 108, 1175 (1957).

[91] M. Bonitz, P. Ludwig, H. Baumgartner, C. Henning, A. Filinov, D. Block, O. Arp, A. Piel, S. Käding, Y. Ivanov, A. Melzer, H. Fehske, and V. Filinov, Physics of Plasmas 15, 055704 (2008).

[92] X. Blanc and M. Lewin, arXiv:1504.01153 [cond-mat] (2015).

[93] F. Perez, L. Gremillet, M. Koenig, S. D. Baton, P. Audebert, M. Chahid, C. Rousseaux, M. Drouin, E. Lefebvre, T. Vinci, J. Rassuchine, T. Cowan, S. A. Gaillard, K. A. Flippo, and R. Shepherd, Physical Review Letters 104, 085001 (2010).

[94] P. K. Patel, A. J. Mackinnon, M. H. Key, T. E. Cowan, M. E. Foord, M. Allen, D. F. Price, H. Ruhl, P. T. Springer, and R. Stephens, Physical Review Letters 91, 125004 (2003).

[95] P. Markowich, C. Ringhofer, and C. Schmeiser, New York 515 (1990).

[96] C. L. Gardner, SIAM J. Applied Math. 54, 409 (1994).

[97] Y. E. Kim and A. L. Zubarev, Physics Letters A 327, 397 (2004).

\section{Appendix A: Derivation of the model and formalism}

In this Appendix, we give the detailed analytical derivation of the equation of state, linearized hydrodynamic theory, dispersion relation, and screened Coulomb potential between fermionic ions over the BCS-BEC crossover in quantum plasma.

\section{Equation of state}

In order to understand the short-range attractive potential and quantum ion-acoustic wave across the BCS$\mathrm{BEC}$ crossover, it is instructive to derive the equation of state in a two-component Fermi gas of electrons and ions of equilibrium density $n_{0}$ for species $\mathrm{s}$, representing electrons $(\mathrm{s}=\mathrm{e})$ or ions $(\mathrm{s}=\mathrm{i})$. In the unitary 
limit, no characteristic length is set by the interparticlepotential since its acoustic wave scattering length $a$ diverges $a \rightarrow \pm \infty$. The energy per particle of a homogeneous two-component Fermi gas at unitary must then depend on the only length characterizing the system (average particle distance, $\left.1 / k_{F}\right)$, with the Fermi wave number $k_{F}=\left(3 \pi^{2} n_{0}\right)^{1 / 3}$.

The chemical potential $\mu_{s}$ is related to the ground state energy per particle $\epsilon_{s}\left(n_{s}\right)$ at zero temperature, $\mu_{s}=\frac{d\left(n_{s} \epsilon_{s}\left(n_{s}\right)\right)}{d n_{s}}$, with density $n_{s}$ and mass $m_{s}$. The extended Thomas-Fermi density functional theory and an analytical fitting formula are used to define $\epsilon_{s}\left(n_{s}\right)$ as follows. First, according to the extended Thomas-Fermi density functional theory [30], $\epsilon_{s}\left(n_{s}\right)$ and $\mu_{s}\left(n_{s}\right)$ at unitary are proportional to those of an ideal Fermi gas,

$$
\epsilon_{s}\left(n_{s}\right)=\frac{3}{5} \xi E_{F s}, \mu_{s}\left(n_{s}\right)=\xi E_{F s},
$$

where $E_{F s}=\hbar^{2} k_{F}^{2} / 2 m_{s}$ ( $\hbar$ is the Plank constant), and $\xi$ is the Bertsch parameter representing the universal character. Existed results for the Bertsch parameter $\xi$ determined by experiments, analytic and simulation calculations are in the range of $[0.2-0.9]$, where $\xi=0.42-0.46$ gives a better fit among these theoretical and experimental results $[30,32,44,62,63]$, and $\xi=1$ represents a noninteracting Fermi gas (ideal Fermi gas). In this work we take $\xi=0.42$ through the whole paper.

\begin{tabular}{cccccc}
\hline \hline & $\alpha_{1}$ & $\alpha_{2}$ & $\alpha_{3}$ & $\beta_{1}$ & $\beta_{2}$ \\
\hline$B C S$ & $\xi$ & $2\left(1-\alpha_{1}\right) / \pi$ & $9 \pi \alpha_{2} / 10$ & 1.4328 & $\alpha_{2} \alpha_{3} \beta_{1}$ \\
$B E C$ & $\xi$ & $2 \alpha_{1} / \pi$ & $54 \pi \alpha_{2} / 25$ & 0.1126 & $3 \alpha_{2} \alpha_{3} \beta_{1}$ \\
\hline \hline
\end{tabular}

Table I. The value of fitting parameters at BCS state and BEC state, respectively.

Second, a reliable analytical fitting formula [32] is used for the BCS and BEC sides,

$$
\begin{aligned}
& \epsilon_{s}\left(n_{s}\right)=\frac{3}{5} E_{F s} f\left(1 / k_{F} a\right), \\
& f\left(1 / k_{F} a\right)=\alpha_{1}-\alpha_{2} \arctan \left(\frac{\alpha_{3}}{k_{F} a} \frac{\beta_{1}+\left|1 / k_{F} a\right|}{\beta_{2}+\left|1 / k_{F} a\right|}\right),
\end{aligned}
$$

which is consistent with the Padé approximation method [31]. The fitting parameters are different between BCS side and BEC side, as shown in Table I. According to the definition of $f\left(1 / k_{F} a\right)$, the chemical potential $\mu_{s}$ can be written in the form of

$$
\mu_{s}=\frac{d\left(n_{s} \epsilon_{s}\left(n_{s}\right)\right)}{d n_{s}}=E_{F s}\left[f\left(1 / k_{F} a\right)-\frac{1}{5 k_{F} a} f^{\prime}\left(1 / k_{F} a\right)\right] .
$$

The nature of fermionic states has significant consequences for the statistical properties of many-particle systems in quantum plasmas. The Pauli exclusion principle forbids identical fermions occupying the same singleparticle state. As a result, the chemical potential of fermionic particles is defined as adding Fermi particles to a system at zero temperature if these particles occupy higher energy levels (assuming that all lower ones are occupied). So it gives rise to a drastic increase of the per-particle energy $\left[\epsilon_{s}\left(n_{s}\right) \propto E_{F s}\right]$ and chemical potential $\left[\mu_{s}\left(n_{s}\right) \propto E_{F s}\right]$ due to $E_{F s} \propto n_{s}^{2 / 3}$ [34]. These formulas enable us to calculate the collective modes at the BCS-BEC crossover by using a quantum hydrodynamic theory at zero temperature. The chemical potential used in the main text is the polytropic equation of state calculated from Eqs. (A1)-(A3), from which one can further obtain analytic expressions for the screened potential and phonon frequency.

\section{Quantum hydrodynamic model}

The low-energy collective dynamics of the superfluid Fermi gas at the BCS-BEC crossover is usually described by the equations of classical superfluid hydrodynamics, which are the time-dependent version of the local density approximation with the Thomas-Fermi energy functional method [30]. Quantum hydrodynamic theory is a generalization of classical superfluid hydrodynamics, including the quantum effects. The quantum hydrodynamic model $[5,37,64-68]$ provides a powerful tool to understand the collective behavior of quantum many-body systems from different points of view, where its dynamical generalization amounts to introducing quantum statistics and diffraction effects into the hydrodynamic equations of classical fluid. The quantum diffraction effect has been proved experimentally in quantum plasmas [69].

We define charge $Z_{s} e$ ( $e$ is the elementary charge), density $n_{s}=\left|\Psi_{s}\right|^{2}$, velocity field $\mathbf{v}_{s}=\frac{\hbar}{i m_{s}} \nabla \frac{\Psi_{s}}{\sqrt{n_{s}}}$, and wave function $\Psi_{s}$, for species $s$. The considered system can be described by the Hamiltonian $[34,37]$ with the electric potential $\phi$,

$$
H_{s}=-\frac{\hbar^{2}}{2 m_{s}} \nabla^{2}+Z_{s} e \phi+\mu_{s}
$$

It is convenient to write the wave function in the form of $[32,34,37]$

$$
\Psi_{s}=\sqrt{n_{s}} e^{i S_{s} / \hbar}
$$

with $m_{s} \mathbf{v}_{s}=\nabla S_{s}$. Here we neglect the effects of entanglement and focus on the collective modes of quantum plasmas. The dynamics of species $s$ at zero temperature can be described by the nonlinear Schrödinger equation

$$
i \hbar \frac{\partial \Psi_{s}}{\partial t}=-\frac{\hbar^{2}}{2 m_{s}} \nabla^{2} \Psi_{s}+Z_{s} e \phi \Psi_{s}+\mu_{s} \Psi_{s} .
$$

By separating Eq. (A6) into its real and imaginary parts, we indeed obtain the equivalent hydrodynamic equations,

$$
\begin{aligned}
& \frac{\partial n_{s}}{\partial t}+\nabla \cdot\left(n_{s} \mathbf{v}_{s}\right)=0, \\
& \frac{\partial \mathbf{v}_{s}}{\partial t}+\frac{\nabla}{m_{s}}\left(Z_{s} e \phi+\mu_{s}+\frac{1}{2} m v_{s}^{2}-\frac{\hbar^{2}}{2 m_{s}} \frac{1}{\sqrt{n_{s}}} \nabla^{2} \sqrt{n_{s}}\right)=0,
\end{aligned}
$$


provided that the fluid is inviscid and irrotational [30]. In the bracket of the second identity, the first term is the electric potential, the second term is the chemical potential, which is regarded as quantum statistical potential due to the Pauli exclusion principle for fermions, the third term is the kinetic potential, and the fourth term is the quantum Bohm potential (quantum pressure) involving the interacting of wave functions due to wave interference $[34,66]$. Indeed, in an ultra-cold quantum plasma, the quantum nature of particles could induce wave properties due to wave interference [12] and Fermi statistics effects due to Pauli exclusion principle [11]. The quantum hydrodynamic equations are closed by the Poisson equation

$$
\nabla^{2} \phi=4 \pi e\left(n_{e}-n_{0}\right)-4 \pi Z_{i} e \delta(r)
$$

Assuming a test ion at position $r=0$ with charge $Z_{i} e$ to realize an analytical form of the electric potential $\phi(r)$ between ions based on Ref. [5].

In order to achieve analytical expressions, which would be critical for understanding the underlying mechanisms of the collective dynamics, the hydrodynamic equations and Poisson equation could be linearized. We apply a linearized fluid description of electrons and ions. We write each quantity as $X=X_{0}+\delta \cdot X_{1}$ where subscript 0 denotes the zero-order constant equilibrium value, and 1 denotes the first-order perturbation. $\delta$ is an ordering parameter for linearization, and has the physical value 1 . This linear method is valid for $X_{0} \gg \delta \cdot X_{1}$. To linearize, we balance all terms in each equation of the same order in $\delta$. The terms involving only subscript- 0 quantities are all order $\delta^{0}$ and must balance, and terms with one subscript-1 quantity are all order $\delta^{1}$ and balance. We treat the velocity field and electric potential as order-1 $\left(\mathbf{v}_{s 0}=0, \mathbf{v}_{s}=\mathbf{v}_{s 1}, \phi_{0}=0, \phi=\phi_{1}\right)$. The hydrodynamic equations and Poisson equation after linearization take the form

$$
\begin{aligned}
& \frac{\partial n_{s 1}}{\partial t}+n_{0} \nabla \cdot \mathbf{v}_{s 1}=0 \\
& \frac{\partial \mathbf{v}_{s 1}}{\partial t}+\frac{\nabla}{m_{s}}\left(Z_{s} e \phi+\mu_{s 1}-\frac{\hbar^{2}}{4 m_{s} n_{0}} \nabla^{2} n_{s 1}\right)=0
\end{aligned}
$$

and

$$
\nabla^{2} \phi=4 \pi e\left(n_{e 1}-Z n_{i 1}\right)
$$

The quantum hydrodynamic model is valid $[5,37,64$, $66,68,70]$ for $\hbar \omega_{e p} \leq E_{F e}$, and the electron-ion collision relaxation time is greater than the electron plasma period, where $\omega_{e p}$ is the electron plasma frequency, given as $\omega_{s p}=\sqrt{4 \pi e^{2} n_{0} / m_{s}}$. The applicability and validity of the quantum hydrodynamic model have been widely tested not only in quantum plasmas $[5,34,37,64-68,70]$, but also in the field of cold atomic systems [30, 32, 62, 71].

\section{Screened potential}

We report a screened electric potential between ions that are shielded by degenerate electrons according to Ref. [5]. In order to obtain an analytical expression of this potential between ions, we assume a test ion at position $r=0$ with charge $Z_{i} e=e$, thus the Poisson equation becomes after linearization

$$
\nabla^{2} \phi=4 \pi e n_{e 1}-4 \pi e \delta(r) .
$$

The linearized density $n_{e 1}(\mathbf{r}, t)$ and velocity $\mathbf{v}_{e 1}(\mathbf{r}, t)$ can be written as

$$
\begin{aligned}
& n_{e 1}(r, t)=n_{e 1} \exp (i(\mathbf{q} \cdot \mathbf{r}-\omega t)) \\
& \mathbf{v}_{e 1}(r, t)=\mathbf{v}_{e 1} \exp (i(\mathbf{q} \cdot \mathbf{r}-\omega t))
\end{aligned}
$$

where $\mathbf{q}$ is the wave vector and $\omega$ is the frequency of acoustic waves. Inserting Eq. (A11) into Eq. (A8), we get

$$
\begin{aligned}
& n_{e 1}=n_{e 0} \frac{\mathbf{q} \cdot \mathbf{v}_{e 1}}{\omega} \\
& \mathbf{v}_{e 1}=i \frac{e}{m_{e} \omega} \nabla \phi /\left(1-\frac{c_{e 0}^{2} q^{2}}{\omega^{2}}-\frac{\hbar^{2} q^{4}}{4 m_{e}^{2} \omega^{2}}\right) .
\end{aligned}
$$

The space Fourier transformation is used for Eq. (A10),

$$
\phi(\mathbf{r})=\iint \frac{d \mathbf{q}}{(2 \pi)^{3}} \phi(\mathbf{q}) e^{i \mathbf{q} \cdot \mathbf{r}} .
$$

Inserting the resultant $n_{e 1}$ from Eq. (A12) into Eq. (A13), leads to the electric potential around an test ion,

$$
\phi(\mathbf{r})=\frac{e}{2 \pi^{2}} \int \frac{\exp (i \mathbf{q} \cdot \mathbf{r})}{q^{2} D} d^{3} q,
$$

where $\mathbf{r}$ denotes the position relative to the position of the test ion. The dielectric constant $D$ and its inverse are given as

$$
\begin{aligned}
& D=1+\frac{\omega_{e p}^{2}}{q^{2} c_{e 0}^{2}+\hbar^{2} q^{4} / 4 m_{e}^{2}}, \\
& \frac{1}{D}=\frac{\left(q^{2} / q_{s}^{2}\right)+\alpha q^{4} / q_{s}^{4}}{1+\left(q^{2} / q_{s}^{2}\right)+\alpha q^{4} / q_{s}^{4}} .
\end{aligned}
$$

Here, $c_{s 0}=\left(\frac{n_{0}}{m_{s}} \frac{d^{2}\left(n_{0} \epsilon_{s}\left(n_{0}\right)\right)}{d n_{0}^{2}}\right)^{1 / 2}$ is the non-interacting sound velocity, $q_{s}=\omega_{e p} / c_{e 0}$ is the inverse Thomas-Fermi screening length, and $\alpha=\hbar^{2} \omega_{e p}^{2} / 4 m_{e}^{2} c_{e 0}^{4}$.

By inserting Eq. (A15) into Eq. (A14) we get

$$
\phi(\mathbf{r})=\frac{e}{4 \pi^{2}} \int\left[\frac{(1+i b)}{q^{2}+q_{+}^{2}}+\frac{(1-i b)}{q^{2}+q_{-}^{2}}\right] \exp (i \mathbf{q} \cdot \mathbf{r}) d^{3} q,
$$

where $q_{ \pm}=\left(q_{s} / \sqrt{4 \alpha}\right)\left[(\sqrt{4 \alpha}+1)^{1 / 2} \pm i(\sqrt{4 \alpha}-1)^{1 / 2}\right]$, and $b=1 / \sqrt{4 \alpha-1}$. With the convolution theorem,

$$
\int \frac{\exp (i \mathbf{q} \cdot \mathbf{r})}{q^{2}+q_{ \pm}^{2}} d^{3} q=2 \pi^{2} \frac{\exp \left(-q_{ \pm} r\right)}{r},
$$


Eq. (A16) yields Eq. (M1) that used in the main text

$$
\phi(r)=\frac{e}{2 r}\left[(1+i b) e^{-q_{+} r}+(1-i b) e^{-q_{-} r}\right],
$$

which corresponds to

$$
\phi(r)=\left\{\begin{array}{l}
\frac{e}{r}\left[\cos \left(q_{i} r\right)+b \sin \left(q_{i} r\right)\right] \exp \left(-q_{r} r\right), \alpha>1 / 4 \\
\frac{e}{r}\left(1+\frac{q_{s} r}{\sqrt{2}}\right) \exp \left(-\sqrt{2} q_{s} r\right), \alpha \longrightarrow 1 / 4 \\
\frac{e}{2 r}(1+i b) \exp \left(-q_{+} r\right) \\
+(1-i b) \exp \left(-q_{-} r\right), \alpha<1 / 4 \\
\frac{e}{r} \exp \left(-q_{s} r\right), \alpha \longrightarrow 0
\end{array}\right.
$$

Here, $r=|\mathbf{r}|$, and

$$
\begin{aligned}
& q_{r}=\left(q_{s} / \sqrt{4 \alpha}\right)(\sqrt{4 \alpha}+1)^{1 / 2}, \\
& q_{i}=\left(q_{s} / \sqrt{4 \alpha}\right)(\sqrt{4 \alpha}-1)^{1 / 2} \\
& \sqrt{1-4 \alpha}=i \sqrt{4 \alpha-1}, \alpha>1 / 4, \\
& \sqrt{4 \alpha-1}=i \sqrt{1-4 \alpha}, \alpha<1 / 4 .
\end{aligned}
$$

\section{Dispersion relation}

The interaction potential between two screened ions is also described by Eq. (A17) [5], by replacing $r$ with the distance between two same ions $r_{m n}=\left|\mathbf{r}_{\mathbf{m}}-\mathbf{r}_{\mathbf{n}}\right|$. Therefore, the interaction potential energy between two screened ions with the same charge $e$ at the positions $\mathbf{r}_{\mathbf{m}}$ and $\mathbf{r}_{\mathbf{n}}$, can be written as

$$
\begin{aligned}
U_{m, n}\left(r_{m n}\right)= & \frac{e^{2}}{r_{m n}} \exp \left(-q_{r} r_{m n}\right)\left[\cos \left(q_{i} r_{m n}\right)\right. \\
& \left.+b \sin \left(q_{i} r_{m n}\right)\right] .
\end{aligned}
$$

We have the motion equation for vibrations of homogeneous lattice ions under the screened Coulomb interactions based on Ref. [5, 72]

$$
m_{i} \frac{d^{2} z_{1 n}}{d t^{2}}=-\sum_{m \neq n} \frac{\partial U_{m n}\left(\mathbf{r}_{m}, \mathbf{r}_{n}\right)}{\partial z_{n}}
$$

where $z_{1 n}=z_{n}-z_{0 n}$ is the vertical displacement of the $n t h$ ion from its equilibrium position $z_{0 n}$. Note that this equation plays a similar role as Eq. (A8) without the chemical potential and quantum pressure of ions.

In order to obtain an analytical dispersion relation, we consider wave oscillations with time and space dependence $X_{1} \propto \exp [i(n L q-\omega t)]$ (frequency $\omega$ and wave number $q) . X_{1}$ represents the first-order perturbation of any physical quantity like $z_{n}, v_{i}$ and $n_{i}$, and $L$ is the nearest neighbor distance. Here, we take $L=d$ determined by $d \phi(r) / d r=0$ at $r=d$ according to the Ref. [5]. If we deal with nearest neighbor screened Coulomb interactions alone according to Refs. [5, 72], dispersion relation without the chemical potential and quantum pressure of ions can be obtained by substituting $z_{1 n} \propto \exp [i(d q-\omega t)]$ into Eq. (A20),

$$
\begin{aligned}
-\omega^{2} m_{i} & =e\left(\frac{d^{2} \phi(r)}{d r^{2}}\right)_{r=d}[\exp (i d q)+\exp (-i d q)-2] \\
& =-4 e\left(\frac{d^{2} \phi(r)}{d r^{2}}\right)_{r=d} \sin ^{2}\left(\frac{q d}{2}\right) .
\end{aligned}
$$

The effect of ion chemical potential and ion quantum pressure can be included in the collective dispersion of quantum ion acoustic waves (phonon excitations), with coupling Eq. (A8), (A17) and (A21) and substituting $v_{i 1}, n_{i 1} \propto \exp [i(n d q-\omega t)]$ into them,

$$
-\omega^{2} m_{i}=-4 e\left(\frac{d^{2} \phi(r)}{d r^{2}}\right)_{r=d} \sin ^{2}\left(\frac{q d}{2}\right)-m_{i} c_{i 0}^{2} q^{2}-\frac{\hbar^{2} q^{4}}{4 m_{i}} .
$$

This Eq. (A22) is rewritten as

$$
\frac{\omega^{2}}{q^{2}}=\frac{e d^{2}}{m_{i}}\left[\frac{d^{2} \phi(r)}{d r^{2}}\right]_{r=d}\left(\frac{\sin (q d / 2)}{q d / 2}\right)^{2}+c_{i 0}^{2}+\frac{\hbar^{2} q^{2}}{4 m_{i}^{2}},
$$

With the help of Eqs. (A21)-(A23), we derive Eq. (M5) that is used in the main text to define the dispersion relation of the quantum ion-acoustic wave with considering the electric potential, chemical potential and quantum pressure of screened ions.

For low wave number $q$ or long wavelength limit $(q \ll$ $\left.k_{F}\right)$ [72], on setting $\sin (q d / 2) \approx q d / 2$, the dispersion relation reduces to

$$
\omega^{2}=\left[\left.\frac{e d^{2}}{m_{i}} \frac{d^{2} \phi(r)}{d r^{2}}\right|_{r=d}+c_{i 0}^{2}\right] q^{2}+\frac{\hbar^{2} q^{4}}{4 m_{i}^{2}} .
$$

Based on Eq. (A24), the ion wave velocity without the ion quantum pressure $\left(\frac{\hbar^{2} q^{4}}{4 m_{i}^{2}}\right)$ is given as

$$
v_{0}=\frac{\omega}{q}=\left[\left.\frac{e d^{2}}{m_{i}} \frac{d^{2} \phi(r)}{d r^{2}}\right|_{r=d}+c_{i 0}^{2}\right]^{1 / 2},
$$

which can be compared to the sound velocity $c_{i 0}$ for noninteracting Fermi gas. The difference is induced from the screened potential between ions and ion quantum pressure, compared to the noninteracting sound velocity $c_{i 0}$. It is necessary to calculate the unitary limit of the sound velocity

$$
\left.c_{s 0}\right|_{k_{F} a \rightarrow \pm \infty}=v_{F s}(\xi / 3)^{1 / 2},
$$

where $v_{F s}=\left(2 E_{F s} / m_{s}\right)^{1 / 2}$. Thus, the roles of linearized model [Eq. (A8)] and the analytical screened potential [Eq. (A17)] are to have a simple analytical expression of the phonon frequencies without the effect of the order parameter $(\Delta$, defined in Eq. (M3) in the main text).

In order to consider the effect of order parameter $(\Delta)$ on the dispersion law of the collective mode for acoustic waves in a paired plasma, we express the dispersion relation based on Ref. [43],

$$
s_{11} s_{22}=\omega^{2} s_{12}^{2},
$$


where

$$
\begin{gathered}
s_{12}\left(\omega, q, \mu_{i}, \Delta\right)=\int_{0}^{\infty} k^{2} d k \int_{0}^{1} d u \frac{1}{E_{+} E_{-}} \frac{E_{+} \eta_{-}+\eta_{+} E_{-}}{\left(E_{+}+E_{-}\right)^{2}-\omega^{2}}, \\
s_{11}\left(\omega, q, \mu_{i}, \Delta\right)=\int_{0}^{\infty} k^{2} d k\left(\int_{0}^{1} d u \zeta_{+}-\frac{1}{E}\right), \\
s_{22}\left(\omega, q, \mu_{i}, \Delta\right)=\int_{0}^{\infty} k^{2} d k\left(\int_{0}^{1} d u \zeta_{-}-\frac{1}{E}\right),
\end{gathered}
$$

$\zeta_{ \pm}=\frac{\left(E_{+}+E_{-}\right)}{E_{+} E_{-}} \frac{E_{+} E_{-}+\eta_{+} \eta_{-} \pm \Delta^{2}}{\left(E_{+}+E_{-}\right)^{2}-\omega^{2}}, \quad E_{ \pm}=$ $\sqrt{\eta_{ \pm}^{2}+\Delta^{2}}, \quad E \quad=\quad \sqrt{\eta^{2}+\Delta^{2}} \quad, \quad \eta_{ \pm}=$ $\left(k^{2} \pm k q u+q^{2} / 4\right) \hbar^{2} /\left(2 m_{i}\right)-\mu_{i}$ and $\eta=k^{2} \hbar^{2} /\left(2 m_{i}\right)-$ $\mu_{i}$. The above equations can be solved numerically, where the MATLAB fsolve function is used to solve the integral equations. The frequency $\omega$ is dependent on $1 / k_{F} a$ through the order parameter [Eq. (M3) defined in the main text] and the chemical potential [Eq. (A3)]. We compare the dispersion relation obtained without [Eq. (A23)] and with [Eq. (A27)] the effect of order parameter $\Delta$ along the BEC-BCS crossover in the main text.

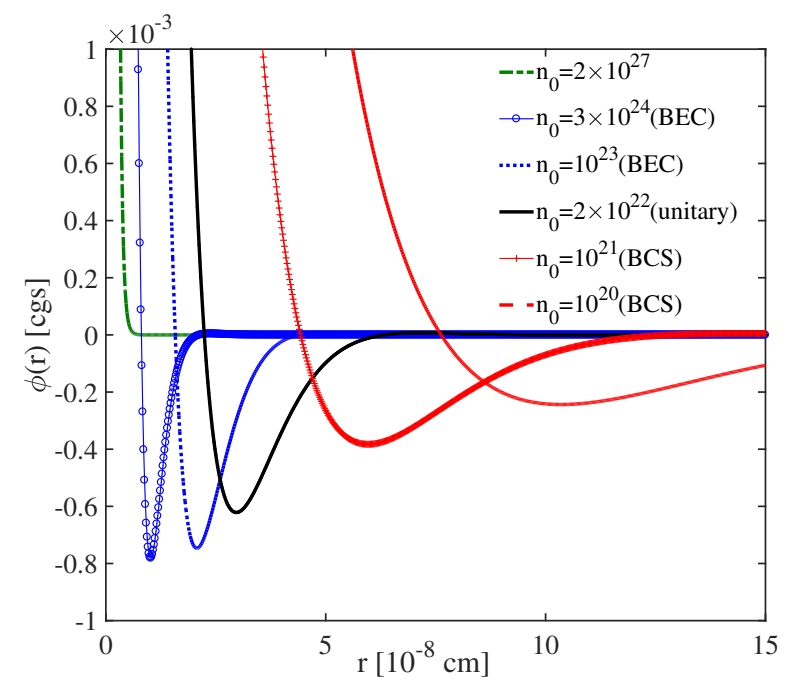

Figure 5. (Color online) The screened potential $\phi(r)$, varying with the plasma density $n_{0}=2 \times 10^{27}(\alpha=0.25), n_{0}=3 \times 10^{24}(\alpha=$ $2.2), n_{0}=10^{23}(\alpha=6.9), n_{0}=2 \times 10^{22}(\alpha=12), n_{0}=10^{21}(\alpha=$ $32), n_{0}=10^{20}(\alpha \approx 70) \mathrm{cm}^{-3}$.

\section{Appendix B: Analysis of the results}

In this Appendix, we discuss the short-range attractive potential between fermionic ions and show the dispersion relation of the quantum ion-acoustic wave, analyze their collective properties (phonon excitations).

\section{Analysis of the short-range attractive potential}

In the following, we calculate the screened potential between two ions in a quantum plasma outside the
BCS-BEC crossover with $n_{0}=2 \times 10^{27}(\alpha=0.25)$ $\mathrm{cm}^{-3}$ and over the BCS-BEC crossover with $n_{0}=3 \times$ $10^{24}(\alpha=2.2), 10^{23}(\alpha=6.9), 2 \times 10^{22}(\alpha=12), 10^{21}(\alpha=$ $32), 10^{20}(\alpha \approx 70) \mathrm{cm}^{-3}$. The range of the BCS-BEC crossover is taken from $n_{0} \approx 10^{15}$ to $n_{0}=3 \times 10^{24}$ $\mathrm{cm}^{-3}$, corresponding to the region of Coulomb bound states [34], which is sufficiently large to probe the presence of the BCS-BEC crossover. The quantum plasma is composed of electrons and ${ }^{4} \mathrm{He}^{+}$ions, with ion mass $m_{i}=6.6 \times 10^{-24} \mathrm{~g}$ and electron mass $m_{e}=9.109 \times 10^{-28}$ $\mathrm{g}$. These values are taken to be the same as in the main text.

In cold atomic gases, the interaction strength between a pair of atoms can be realized by magnetically tuned scattering resonances, named as Feshbach resonances[1618], offering the unique possibility to realize the BCSBEC crossover. The Feshbach resonance was shown in Ref. [39] with infinite scattering length at the threshold (called the unitary point) for the formation of a bound state. Once this bound state is formed, the scattering length changes sign $(-\infty \longrightarrow+\infty)$, and the magnitude decreases with increasing the strength of potential.

The BCS-BEC crossover from Cooper pairs to molecules appears when the size of molecules is of the order of the inter-particle spacing $\propto 1 / k_{F}$ in atomic systems [32]. It is interesting to explore whether this phenomenon also happens for the cold ion system due to the screened potential between a pair of ions. Although the screened Coulomb interactions make the physics different in a cold plasma, compared to a cold atomic system, the BCS-BEC crossover is expected to occur in a cold plasma. We tried to understand the mechanism for forming the BCS-BEC crossover in a cold plasma, where the inter-ion potential and phonon excitations (acoustic waves) might be essentially different from those of atomic systems at the BCS-BEC crossover.

We first try to answer how the screened potential changes its behavior with the plasma density $n_{0}(\alpha)$ as it was done in Ref. [5]. Parameter $\alpha$ represents the importance of the quantum recoil effect, as it is the coefficient of the quantum pressure term [see the terms of $\sim q^{4}$ in Eq. (A15)]. Here $\alpha=\hbar^{2} \omega_{e p}^{2} / 4 m_{e}^{2} c_{e 0}^{4}$ is dependent on the density $n_{0}$, through the electron plasma frequency $\omega_{e p}$ and the noninteracting sound velocity $c_{e 0}$ determined by the electron chemical potential $\mu_{e}$, which are self-consistently included in the expression of $\phi(r)$ [Eqs. (A15)-(A17)].

As it has been reported in Ref. [5] that, the screened potential shows negative values for $\alpha>0.25$, the effect of $n_{0}$ on the potential $\phi(r)$ as a function of $r$ is drawn in Fig. 5, with several values of $n_{0}(\alpha)$. It is clearly seen that the potential shows both short-range positive and negative values, indicating the short-range repulsive and attractive interactions between two ions with different distance. The negative potential value increases while their distance decreases, as shown in Fig. 5, which is expected for realizing the BCS-BEC crossover. The value of minimum potential $\phi(r)$ at different distance $r$ 
is shown in Table II for different $n_{0}(\alpha)$. Note that the different regimes depend on the potential. As shown in Fig. 5 and Table II, the deep BEC state is achieved tuning $n_{0}=3 \times 10^{24} \mathrm{~cm}^{-3}\left(\phi(r)=-7.8 \times 10^{-4}[\mathrm{cgs}]\right)$. The unitary regime occurs when $n_{0}=2 \times 10^{22} \mathrm{~cm}^{-3}$ $\left(\phi(r)=-6 \times 10^{-4}[\mathrm{cgs}]\right)$. We can obtain the deep BCS of the ions when $n_{0}=10^{20} \mathrm{~cm}^{-3}\left(|\phi(r)|=2.4 \times 10^{-4}\right.$ $[\mathrm{cgs}])$. We indeed see the short-range attractive potential that agrees with the Lennard-Jones-type potential in the range of $10^{-8} \leq r \leq 10.5 \times 10^{-8} \mathrm{~cm}$, which is on the order of average ion distance $n_{0}^{-1 / 3}$. This is just the condition required for the BCS-BEC crossover.

\begin{tabular}{lcccccc}
\hline \hline$n_{0} \mathrm{~cm}^{-3}$ & $10^{20}$ & $10^{21}$ & $2 \times 10^{22}$ & $10^{23}$ & $3 \times 10^{24}$ & $2 \times 10^{27}$ \\
\hline$\phi(r) \times 10^{4}[\mathrm{cgs}]$ & -2.4 & -3.8 & -0.6 & -7.5 & -7.8 & 0 \\
$r \times 10^{8}[\mathrm{~cm}]$ & 10.5 & 5.8 & 3 & 2.1 & 1 & 0.6 \\
\hline \hline
\end{tabular}

Table II. The value of attractive potential $\phi(r) \times 10^{4}[\mathrm{cgs}]$ between a pair of ions at different distance $r \times 10^{8}[\mathrm{~cm}]$ for several values of $n_{0}$ across the BCS-BEC crossover (BCS: $n_{0}<$ $2 \times 10^{22}$, unitary: $n_{0}=2 \times 10^{22}$, BEC: $2 \times 10^{22}<n_{0} \leq 3 \times 10^{24}$ ) and outside the crossover $\left(2 \times 10^{27}\right)$.

The minimum value of the potential occurs at $r=10^{-8}$ $\mathrm{cm}$ (deep BEC), $r=3 \times 10^{-8} \mathrm{~cm}$ (unitary), and $r=$ $1.05 \times 10^{-7} \mathrm{~cm}$ (deep BCS), as shown in Table II, indicating that the range of attractive potential gets smaller from deep BCS $\left(\sim 10^{-7} \mathrm{~cm}\right)$ to deep BEC $\left(\sim 10^{-8} \mathrm{~cm}\right)$ states. The attractive potential reaches its minimum value in the deep BEC region and is almost unchanging with further increasing $n_{0}$. This is consistent with the prediction that the strongest attraction is resolved by dimer formation, while a repulsion occurs between dimers with further decreasing the distance [39]. Indeed only short-range repulsive potential exists for small enough distance $r<6 \times 10^{-9} \mathrm{~cm}$ with large enough density $n_{0} \geq 2 \times 10^{27} \mathrm{~cm}^{-3}(\alpha \leq 0.25)$ as shown in Fig. 5 .

It is expected that the short-range attractive potential can form fermionic ion pairs, which in fact may collapse into a BEC state. The short-range attractive potential with adjustable strength agrees on the role of the Feshbach resonance, preferable for the realization of BCSBEC crossover in a cold plasma without a magnetic field. In other words, the screened ions can be trapped in the negative part of the exponential oscillating potential [see Eq. (A17) ], which leads to ion clusters (like molecules) depending on the plasma density $n_{0}$. The potential $\phi(r)$ takes its negative value is allowed by the combined effects of the electron quantum statistical potential and the electron quantum Bohm potential at zero temperature [5]. Physically, this involves the overlapping of electron wave function due to the Heisenberg uncertainty and Fermi statistics effect due to Pauli exclusion principle $[11,12,34,66]$, leading to a short-range negative hard core electric potential. This qualitative behavior has been reported by Shukla and Eliasson [5]. Thus, the BCS-BEC crossover is realized by the short-range attractive potential between a pair of ions, which exhibits new insight into quantum plasmas.

The distance where the potential takes minimum value is defined as the nearest neighbor ion distance and noted as $d$, which is essential to compare the range of inter-ion potential and average ion distance. For this purpose, we seek the solution of relation $\frac{\partial \phi(r)}{\partial r}=0$, from which we determine and plot $d$ in Fig. 6(a), the minimum potential $\phi(d)$ in Fig. 6(b) and the Laplacian of the potential $\left.\frac{\partial^{2} \phi(r)}{\partial r^{2}}\right|_{r=d}$ in Fig. 6(c) at $r=d$, and the acoustic wave period $\sin ^{2}(q d / 2)$ under this potential in Fig. 6(d), as a function of $1 / k_{F} a$.

The value of $d$ decreases linearly with increasing $1 / k_{F} a$ in the BCS region, then rapidly drops at unitary, and decreases more slowly in the BEC region with increasing $1 / k_{F} a$, as shown in Fig. 6(a). These features are consistent with Fig. 5. In general, the distance $d$ decreases through the BCS-BEC crossover, which is just the phenomenon that particle distance decreases over the BCSBEC crossover, reported in Refs [32, 40, 62]. We believe that the variation of distance $d$ between two ions is evidence of the formation of ion pairs at BEC, resulting in a variety of new features in quantum plasmas.

At $r=d$, the potential $\phi(d)$ indeed take negative values, as shown in Fig. 6(b), where its strength increases over the BCS-BEC crossover, leading to the formation of ion pairs or ion clusters (analogous to dimers) in the BEC region. This negative Lennard-Jones-like potential is relevant to the ion-binding potential in the ultracold plasma. The binding potential is determined by the depth of the minimum potential $\phi(d)$. Correspondingly, the value of $\phi^{\prime \prime}(d)$ increases through the BCS-BEC crossover, as seen from Fig. 6(c), which further determines the acoustic wave frequency and the character of dispersion relation [see Figs. 7-9 below]. Note that, $\phi(d)$ and $\phi^{\prime \prime}(d)$ take finite negative values and positive values, respectively, over the whole BCS-BEC crossover, indicating that there always occurs attractive potential between a pair of screened ions at a proper distance for $10^{15} \geq n_{0} \leq 3 \times 10^{24} \mathrm{~cm}^{-3}\left(3 \times 10^{4} \geq \alpha \geq 2\right)$. The value of $2 \pi / k_{F} d$ is plotted in Fig. 6(d), which is the period of oscillating frequency [see Eq. (A23) and Figs. 7-9 below]. The period increases with increasing $1 / k_{F} a$, taking values of $0-2.5$ at BCS side, 2.5 at unitary, and $2.5-7.5$ at BEC side.

\section{The dispersion relation of acoustic waves}

This subsection discusses the dispersion relation of quantum ion-acoustic waves for unpaired ions since intraspecies of the ions may not form pairs but contribute to phonon excitations. Note that the phonon frequency for paired ions is shown in the main text. The energy per particle $\epsilon_{s} / E_{F s}$, chemical potential $\mu_{s} / E_{F s}$, and sound velocity $c_{s 0} / v_{F s}$ are plotted as a function of $1 / k_{F} a$ in Fig. $7(\mathrm{a})$ and $n_{0}$ in Fig. $7(\mathrm{~b})$, across the BCS-BEC crossover. The parameter $1 / k_{F} a$ characterizes the different interaction regimes and the interaction strength 

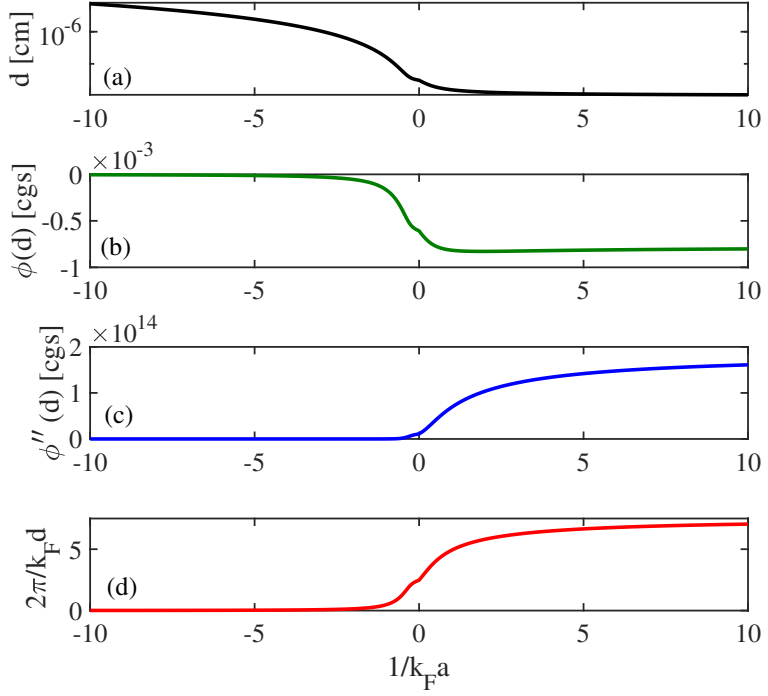

Figure 6. (Color online) (a) The distance $d$ defined by $\frac{\partial \phi(r)}{\partial r}=0$, (b) the potential $\phi(d)$, (c) its second derivative $\phi^{\prime \prime}(d)$ at $r=d$, and (d) the acoustic wave period $2 \pi / k_{F} d$ under the potential, as a function of $1 / k_{F} a$, over the BCS-BEC crossover.

increases with increasing $1 / k_{F} a$ (through increasing $n_{0}$ ). The calculation of energy per particle, chemical potential and sound velocity are based on Eqs. (A1)- (A3) and the results agree well with that in Refs. [32, 40, 62]. At unitary at $1 / k_{F} a=0\left(n_{0}=2 \times 10^{22} \mathrm{~cm}^{-3}\right)$, the sound velocity is calculated from $c_{s 0}=v_{F s}(\xi / 3)^{1 / 2}$. In both deep BCS and BEC states, the energy per particle, chemical potential, and sound velocity take asymptotic limits and are almost independent of $1 / k_{F} a$. However, at unitary, the curves show a rapid decrease with increasing $1 / k_{F} a\left(n_{0}\right)$, as shown in Fig. $7(\mathrm{a}, \mathrm{b})$, indicating the formation of the BCS-BEC crossover. One sees the crossover from the BCS $\left(1 / k_{F} a=-10, n_{0}=10^{15} \mathrm{~cm}^{-3}\right)$ to unitary $\left(1 / k_{F} a=0, n_{0}=2 \times 10^{22} \mathrm{~cm}^{-3}\right)$ then to BEC $\left(1 / k_{F} a=10, n_{0}=3 \times 10^{24} \mathrm{~cm}^{-3}\right)$, as shown in Fig. 7 , which suggests that the BCS-BEC crossover is indeed a continuous transition.

The dispersion relation [see Eq. (A23)] is not only dependent on the electron chemical potential and electron quantum pressure, included in the value of $\left.\frac{\partial^{2} \phi(r)}{\partial r^{2}}\right|_{r=d}$, but also relevant to the ion chemical potential [behaves as the second term $c_{i 0} q^{2}$ in Eq. (A23)] and the ion quantum pressure [behaves as the third term $\propto q^{4}$ in Eq. (A23)]. For the purpose of understanding the collective properties of quantum ion-acoustic waves at the BCS-BEC crossover, the phonon frequency is obtained for all the possible values of $1 / k_{F} a$ [varying through the plasma density $n_{0}$ (see Fig. $3(\mathrm{~d})$ in the main text)]. We plot the phonon frequency as a function of $1 / k_{F} a$ in Fig. $7(\mathrm{c})$ and $n_{0}$ in Fig. $7(\mathrm{~d})$, for different $q$.

The frequency presents slowly changing acoustic mode at the BCS side, and a sharp increase around the unitary point, then a significantly slower increase at the BEC side, as shown in Fig. 7(c,d). In the regime of a strongly interacting unitary Fermi gas, an abrupt change in the phonon frequency occurs, which may be a signature for a transition from BCS to BEC phase. This phenomenon agrees with the evolution of the Anderson-Bogoliubov damped mode in the BCS limit to the Bogoliubov sound mode in the BEC limit [73], predicted by Melo et al. [73] based on the time-dependent Ginzburg-Landau equation.

The frequency dependence on $n_{0}$ is consistent with that on $1 / k_{F} a$, since $1 / k_{F} a$ is determined by the plasma density $n_{0}$ [see Fig. $7(\mathrm{~d})$ in the main text]. This is qualitatively agreeing with the breathing modes in atomic systems obtained by various theoretical methods such as beyond mean-field theory [74], hydrodynamic method [32] and time-dependent density functional theory [62]. Because the short-range attractive potential between a pair of ions in cold plasmas plays the role of an external trapped potential in atomic systems. The frequency is inversely proportional to the chemical potential, and its dependence on $1 / k_{F} a$ is inverted compared to the chemical potential and the non-interacting sound velocity $[32,40,62]$. The frequency increases with increasing $1 / k_{F} a$, indicating a positive-shift of the frequency with increasing the interaction strength, which coincides with the feature predicted by Refs. [32, 40, 62, 74].
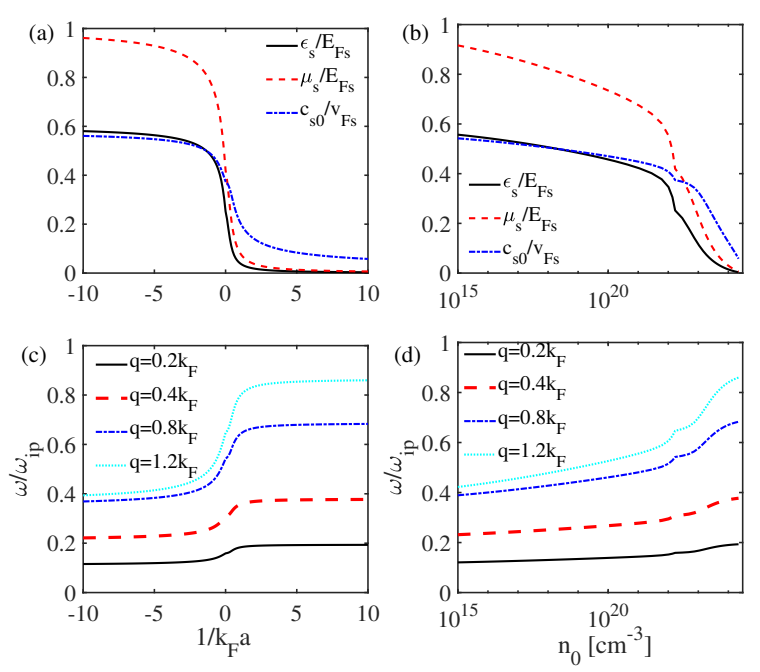

Figure 7. (Color online) The energy per particle $\epsilon_{s} / E_{F s}$ (solid line), chemecal potential $\mu_{s} / E_{F s}$ (dashed line), and sound velocity $c_{s 0} / v_{F s}$ (dash-dotted line) as a function of $1 / k_{F} a(\mathrm{a})$ and $n_{0}(\mathrm{~b})$. Phonon frequency $\omega / \omega_{i p}$ for $q=0.2 k_{F}$ (solid line), $q=0.4 k_{F}$ (dashed line), $q=0.8 k_{F}$ (dash-dotted line), $q=1.2 k_{F}$ (dotted line) as a function of $1 / k_{F} a(\mathrm{c})$ and $n_{0}(\mathrm{~d})$.

We perform systematic calculation and detailed discussion on the phonon frequency $\omega / \omega_{i p}$ dependence on $1 / k_{F} a$ for understanding the collective property of cold plasmas at the BCS-BEC crossover. The frequency dependence on $1 / k_{F} a$ for different wave number $q$ is shown 
in Fig. 8. For small $q$, the frequency is lower at BCS state, while it rises quickly at unitary, and it is higher at BEC state, which is consistent with the profile of $\left.\frac{\partial^{2} \phi(r)}{\partial r^{2}}\right|_{r=d}$ [see Fig. 6(c) above]. The frequency dependence on $1 / k_{F} a$ is contrast to the profiles of $\mu_{i}$ and $c_{i 0}$ [see Fig. $7(\mathrm{a}, \mathrm{b})$ above], as the term $\left.\propto \frac{\partial^{2} \phi(r)}{\partial r^{2}}\right|_{r=d}$ is dominant compared to the other terms [see Eq. (A23)] for small $q$. Moreover, the frequency first decreases in the BCS region, then increases and approaches its maximum value in the BEC region, passing through the minimum near unitary point $1 / k_{F} a=0$, indicating nonmonotonic behavior at $q=2.5 k_{F}$. This behavior agrees phenomenologically with that in an atomic system in Ref. [32] over the BCS-BEC crossover.

With increasing $q$, the frequency minimum changes to the maximum, and $\omega / \omega_{i p}$ first increases then decreases over the crossover in the presence of $\sin ^{2}(q d / 2)$ [see Eq. (A23)], as shown in Fig. 8(c). With further increas$\operatorname{ing} q$, the frequency peak divides into a valley and a peak, with the minimum and maximum occurring close to the left and right of the unitary point, respectively, as shown in Fig. 8(d). This result is ascribed to the presence of $\sin ^{2}(q d / 2)$ due to the screened potential, yielding an oscillating frequency concerning $q$ [see Fig. 9 below].

The phonon frequencies reach the asymptotic values in the deep BEC limit, indicating an asymptotic behavior, which agrees with the character of the breathing modes in a trapped atomic system [32]. A local maximum occurs near the unitary point in the BCS regime at a decent wave number $q$, indicating the nonmonotonic character of the phonon frequency. The experimental measurements of phonon frequency confirm this result in a trapped atomic system, where the radial frequency has shown an abrupt decrease with increasing $1 / k_{F} a$ in the regime of BCS near the unitary point [23]. This experiment also predicted a negative-shift of the radial frequency [23] with increasing $1 / k_{F} a$, i.e., increasing the interaction strength, with a spin mixture of trapped ${ }^{6} \mathrm{Li}$ atoms in a cigar-shaped molecular BEC confined by a laser beam. Although this is contrast to our results for small $q$ as shown in Fig. 8(a,b), it has shown consistent features for a proper large $q$ [see Fig. 8(c,d)]. The experimental data [23] confirms our results for a good wave number. i.e., the abrupt change and negative shift of the phonon frequency occur in nearly the same way [see Fig. 8(c,d)]. This further confirms the occurrence of the BCS-BEC crossover in cold plasmas, which is guaranteed by the short-range attractive potential.

Are these different phonon frequencies across the BCSBEC crossover not pure fantasy? There are strong reasons to believe that this is a very realistic scenario at the BCS-BEC crossover examined in ultra-cold quantum plasma. Indeed, our theoretical results on the phonon frequency in a cold quantum plasma show three possible reasons for realizing the BCS-BEC transition, compared with the collective mode in the trapped atomic system with reasonable choices of the relevant parameters. The corresponding features, could be confirmed on the basis of available theoretical models [32, 40, 62, 74] and experimental measurements [23] over the BCS-BEC crossover with three reasons. First, the cold atom is trapped externally by a magnetic field or laser in the atomic system; in comparison, the cold ion is trapped in the negative part of screened potential by the short-range attractive interactions. Second, the phonon frequency exhibits the same dependence on $1 / k_{F} a$ as that of atomic gas, although the values of them differs in the two cases. Third, both systems have nonmonotonic and asymptotic characters and abrupt changes at and near the unitary point.
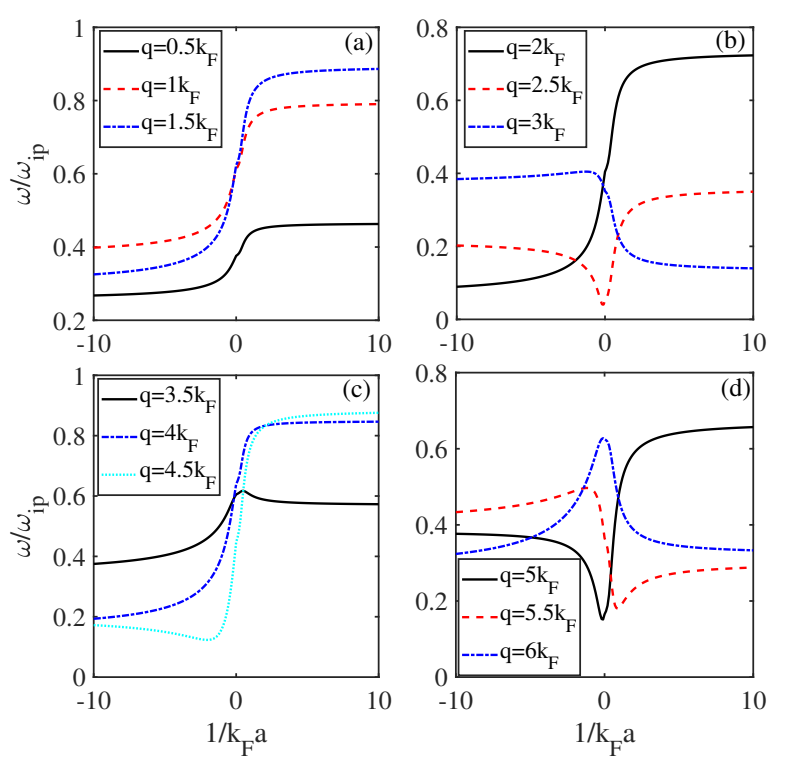

Figure 8. (Color online) Phonon frequency $\omega / \omega_{i p}$ as a function of $1 / k_{F} a$ for different $q$.

The dispersion relation is shown to illustrate the effect of $1 / k_{F} a$ in Fig. $9(\mathrm{a}, \mathrm{b}, \mathrm{c})$, and the contour of $\omega / \omega_{i p}$ as functions of $1 / k_{F} a$ and $q$ in Fig. 9(d). A dramatic character of Fig. 9 is the persistence of linear frequency dependence on $q$ as long as $q \leq k_{F}$, which agrees well with the classical ion-acoustic wave dispersion $\left(\omega=v_{0} q\right)$ in the limit of small Debye length and low-frequency oscillations, where $v_{0}$ is the wave speed in a plasma. Otherwise, with increasing $q$ the curves start to oscillate with a period of $2 \pi / k_{F} d$ [see Fig. $6(\mathrm{~d})$ above], due to the effect of term $\sin ^{2}(q d / 2)$. This guarantees the existence of periodic oscillating frequency. The frequency almost takes a periodic oscillation for small $q\left(q / k_{F} \leq 5\right)$, where the maximum value is determined by $\left.\frac{\partial^{2} \phi(r)}{\partial r^{2}}\right|_{r=d}$, smaller than ion plasma frequency $\omega_{i p} \approx 2 \times 10^{10}-10^{15} \mathrm{~s}^{-1}$ over the BCS-BEC crossover.

The frequency profile at different $1 / k_{F} a$ qualitatively agrees with each other, but the value is different at BEC, unitary, and BCS states. The amplitude of the oscillating frequency is highest at BEC, higher at unitary, and high at BCS state since this amplitude increases with 
increasing the plasma density. The three curves separate from $q=0$, and their difference first becomes larger then smaller with increasing $q$ due to the periodic property. Our results give the nontrivial effect of the parameter $1 / k_{F} a$ on the phonon dispersion for small $q$, due to Eq. (A23). Indeed, for large $q\left(q \gg k_{F}\right)$, the frequency increases more quickly with increasing $q$, and finally merges and shows a quadratic $q$ dependence due to the dominant effect of the third term $\sim \hbar^{2} q^{4} / 4 m_{i}^{2}$, compared to the first and second terms in Eq. (A23).

As expected, the frequency is nearly the same at unitary $\left(1 / k_{F} a=0\right)$ and BEC state $\left(1 / k_{F} a=0.1\right)$, while it is a bit lower at BCS state $\left(1 / k_{F} a=-0.1\right.$ ), as shown in Fig. 9(a). Because the frequency varying with $1 / k_{F} a$ is mainly determined by $\left.\frac{\partial^{2} \phi(r)}{\partial r^{2}}\right|_{r=d}$ [see Eq. (A23) and Fig. 6(c) above], which takes similar values at $1 / k_{F} a=0$ and $1 / k_{F} a=0.1$, but slightly smaller value at $1 / k_{F} a=-0.1$. The three curves separate more significantly with increasing the absolute value of $1 / k_{F} a$, especially for small $q$, as shown in Fig. 9(b,c). Whereas their difference gets smaller with increasing $q$ due to the term $\sim \hbar^{2} q^{4} / 4 m_{i}^{2}$ in Eq. (A23), finally they merge, and the oscillation vanishes for $q \geq 20 k_{F}$. The frequency oscillation disappears more quickly for the BCS state than that for the BEC state, and the unitary case is moderate between them, which are determined by the strength of short-range attractive potential [see Fig. 6(b) above].

The frequency dependence on $q$ is quasi-periodic through the BCS-BEC crossover, with a period of $2 \pi / k_{F} d$, as shown in Fig. 9(d). The frequency reaches the asymptotic value in the deep BEC and shows nonmonotonic behavior on $1 / k_{F} a$ for a larger wave number. These results qualitatively agree with the experimental measurements of phonon frequency in a trapped atomic system [23], which further confirms the occurrence of the BCS-BEC crossover in the cold quantum plasma. However, the physical mechanism is different for the two systems.

\section{Appendix C: Possible comparison between cold ion and atomic systems over the BCS-BEC crossover}

In this Appendix, we discuss the possible comparison between cold ion and atomic systems over the BCS-BEC crossover. Quantum plasma is easily found in nature like the cores of giant planets and white dwarf stars [34, 7577], due to the high density (greater than solid-state density $\sim 10^{23} \mathrm{~cm}^{-3}$ ) caused by gravitation. It has been indicated that electrons are expected to be quantum degenerate in the core of the Sun [75]. Quantum plasma also occurs in various modern laboratories, using shock waves [78], pinches in high-current-carrying plasmas [79, 80], gas guns [81], laser beams and ion beams $[82,83]$. A different kind of quantum plasma occurs in metals and semiconductors, where the valence electrons behave as quasi-free, characterized by a Fermi gas. Moreover, strong compression of a atomic (molecu-
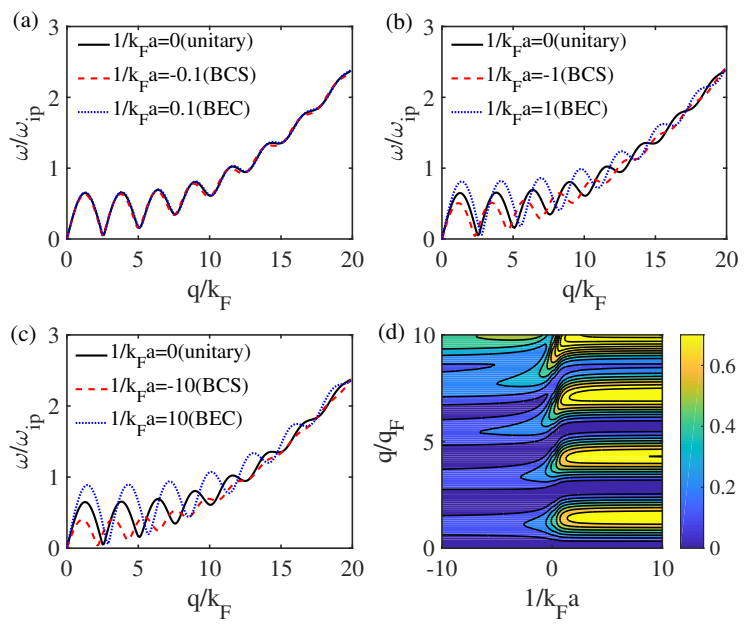

Figure 9. (Color online) Phonon frequency $\omega / \omega_{i p}$ as a function of $q$ for different $1 / k_{F} a$ over the BCS-BEC crossover (a,b,c), and the contour of $\omega / \omega_{i p}$ as functions of $1 / k_{F} a$ and $q(\mathrm{~d})$.

lar) gas could transforms matter from a neutral state of atoms and molecules into a fully ionized two-component quantum plasma with electrons and ions, where bound states may exist at low temperature and high density. Correspondingly, the quantum Coulomb coupling parameter (Brueckner parameter) is $r_{s} \sim \frac{1}{k_{F} a_{B s}} \propto \frac{|e \phi(d)|}{E_{F s}}$. i. e., $r_{e} \approx 1, r_{i} \approx 10^{4}$ with $n_{0} \sim 10^{22} \mathrm{~cm}^{-3}$ at zero temperature [34]. Here $a_{B s}=\frac{\hbar^{2}}{m_{s} e^{2}}$ is the effective Bohr radius. Finally, ultra-dense quantum plasmas could exist in nuclear matter, i. e., quark-gluon plasma (QGP) and the Big Bang, achieved by continued compression of the quantum plasma of electrons and nuclei, where details of the interactions are complex but dominated by Coulomb interactions [84-86].

Previous hydrodynamic theories of the BCS-BEC crossover mainly focused on neutral gas in an atomic system $[30,32,62,71]$. Our theoretical results for ion pairs in ultracold plasmas could be equivalently important as atom pairs for a BCS-BEC crossover. The fact is that atoms like ${ }^{4} \mathrm{He}$ considered in atomic systems [32] can be treated as an ion sphere-forming of the nucleus and electrons as a charged cloud surrounding the nucleus. The dense Thomas-Fermi atomic gas [30] is analogously replaced by a dense Thomas-Fermi plasma [5, 34], where the ions are shielded by nonrelativistic degenerate electrons at zero temperature. The shielding radius of the sphere is defined as the Debye radius or Thomas-Fermi radius $[34,64,66,70] \lambda_{F}=\left(E_{F e} / \omega_{e p}\right)^{1 / 2} \propto 1 / k_{F}$, which is the same order with the inter-particle spacing in atomic unitary systems. Thus, the range of inter-particle potential comparable with inter-particle spacing in the plasma systems, is similar to those in atomic systems, where the Fermi gas exhibits universal properties and is regarded as unitary Fermi gas, irrelevant to neutral or charged gas.

The theoretical model may be realized experimentally 
by using a 3D solid like ${ }^{4} \mathrm{He}$ including ${ }^{4} \mathrm{He}^{+}$ions and electrons. Sound waves with dispersion relation $c_{s 0} q$ are pressure waves propagating from one layer to the next by collisions among the atoms or molecules. In an electrons and ions system, with no neutrals and few collisions, a consistent phenomenon occurs, called an ion acoustic wave (phonon excitation), or, simply, an ion wave. Acoustic waves can occur through the intermediary of an electric field. Experimental verification of the existence of acoustic waves was first accomplished by Wong et al. [87, 88], where Q-machine experiment was designed to detect waves and wave velocities [87,88].

In discussion, the BCS-BEC crossover between ultracold plasma and atomic gas can be directly compared, where differences appear only in the interaction potential. An external trapped potential is applied for the atomic system, where a Feshbach resonance controlled by a magnetic field is used to tune the s-wave scattering length and the interaction potential between two fermionic atoms in a cold atomic system. The short-range attractive potential between fermionic ions screened by degenerate electrons plays the role of effective interaction potential in a cold ion system. This is a controllable interaction, where interaction range and magnitude could be controlled by varying the plasma density.

Indeed, we obtain short-range attractive potential between a pair of screened ions with increasing strength from BCS to BEC as the potential range decreases, which agrees with the main principle of BCS-BEC crossover. With the presence of short-range attractive potential at scales (average ion distance $\sim 1 / k_{F}$ ), the ions can be trapped in the negative part of exponential oscillating screened potential [see Eq. (A17)]. This leads to the formation of ion pairs or ion clusters, depending on the plasma density $n_{0}$, realizing the BCS-BEC crossover, in the field of strongly coupled quantum plasmas [34, 4751].

The s-wave scattering length indeed can be tuned from $-\infty$ to $+\infty$ due to the short-range attractive potential. This guarantees the existence of BCS-BEC crossover in cold ion systems. Here bound pairs are composed of fermionic ions of a different spin. As a result, the system, originally a Fermi gas, is transformed into a bosonic gas of ion-ion pairs. The possibility of tuning the scattering length across the BCS-BEC crossover from negative to positive values can provide a continuous connection between the physics of Fermi superfluidity and BEC state, including the strongly interacting unitary Fermi gas at the middle of the crossover. Moreover, it is shown that the resulting quantum ion-acoustic waves exhibit distinct dispersion relations across the BCS-BEC crossover, which provide a direct route to probe the changing scattering length, as well as the BCS and BEC states. It would deserve an experimental measurement of a phonon dispersion [89] varying continuously from BCS to BEC state.

It is important to note that the short-range attractive potential is an elegant mechanism of pure electron collec- tive quantum behavior, and it vanishes in the quantum limit $\hbar \longrightarrow 0$. In quantum mechanics, the spin configuration of two fermions can be antiparallel or parallel, where the energy of an antiparallel state is lower than the parallel state, and this antiparallel state is named the ground state, which can interact with s-wave. We show that the mechanism of the s-wave interaction is characterized by the low-energy collisions between two fermionic ions when the interaction range is on the same order of the inter-ion spacing, which permits the ions to undergo a BCS-unitary-BEC crossover. Therefore, the bounded ${ }^{4} \mathrm{He}^{+}$ions with the s-wave interaction over the BCS-BEC crossover are virtually occupied in pairs of opposite spin. This is analogous to the paired electrons with opposite spin in the ground state of a superconductor [90]. Indeed, the Pauli exclusion principle states that two fermions in a bounded state cannot have the same quantum numbers, and only two fermions can occupy the same orbital where they must have opposite spin states.

It is necessary to mention that there are situations where fermions manage to transform into bosons in order to achieve lower energy, i.e., the particles are favorable to settle at the position of minimum attractive potential. This principle is the same as that for Cooper pair formation where two fermions form a weakly bound pair which obeys Bose statistics, which is possible due to the short-range attractive potential between a pair of ions. Such fermionic ion pairs, like electron Cooper pairs, may be responsible for the loss of electrical resistance, i. g., superconductivity, in various materials.

We realize the BCS-BEC crossover for fermionic ion pairs in ultra-cold quantum plasmas in the presence of short-range attractive potential due to the quantum statistics and diffraction effects of degenerate electrons at zero temperature, offering a new platform to quantum simulate the BEC-BCS crossover in cold quantum plasma. This may open new stimulating perspectives in the interdisciplinary region of cold plasma and atomic system. From the many-body point of view, the study of BCS-BEC crossover in an ultra-cold plasma opens a different application area and richer class of questions in the interdisciplinary region of cold quantum plasma and cold atomic systems.

This work based on the generalized hydrodynamic theory, further confirms the existence of the Lennard-Joneslike short-range attractive potential and its inevitable role in the formation of ion pairs over the BCS-BEC crossover, which might be important for plasma crystallization $[8,13,34,91,92]$. and strong laser-matter interaction in laboratory [83, 93, 94]. Hydrogen and helium were predicted to form stable crystals controlled by the carrier density [13], which should be realized experimentally with laser or ion beam techniques. Our results confirm that the strength and range of the short-range attractive potential can indeed be tuned by the density of plasma, which may be one of the essential factors yielding strong ion-coupling in planets. The introduction of such short-range attractive potential, which brings the 
ions closer together might also lead to breakthroughs in supercomputing, semiconductor, and nanotechnology sciences [8, 52, 95-97]. 